\title{
Mesure des populations de processionnaire du pin (Thaumetopoea pityocampa Schiff - Lepidoptere Thaumetopoeidae) au Mont-Ventoux
}

\author{
C. GERI * et C. MILLER ** \\ avec la collaboration technique de D. XEUXET \\ * I.N.R.A., Station de Recherches de Zoologie et de Bioconotique forestières \\ Centre de Recherches forestieres d'Orléans, Ardon, F 45100 Olivet \\ *: I.N.R.A.-C.R.Z., Mission informatique \\ F 78350 Jouy-en-Josas
}

\begin{abstract}
Résumé
L'article est consacré à la transposition aux reboisements du Mont Ventoux (Vaucluse) des méthodes de recensement des populations de processionnaires, par comptage des nids, mises au point dans les forêts d'altitude de la Corse.

Les populations sont dénombrées suivant un plan d'échantillonnage au hasard stratifié dans une zonc de 320 ha, située à l'extrémité occidentale du massif. La précision des estimations est discutée. Des analyses statistiques multidimensionnelles (étude des corrélations, analyses de variance et de covariance non orthogonales, analyses factorielles en composantes principales, analyses discriminantes) sont effectuées pour étudier les relations entre les populations et leur cnvironnement physique et forestier, apprécier l'impact d'opérations de traitement et rendre compte de l'incidence de certains facteurs intervenant dans la dynamique des populations de l'insecte.

L'intérêt de ces différentes analyses statistiques est comparé. Le dispositif d'échantillonnage permet d'apprécier les fluctuations naturelles des populations et met en évidence l'efficacité d'un traitement au diflubenzuron. La comparaison des estimations représentatives du massif, et de dénombrements de nids effectués dans 3 parcelles par l'Office National des Forêts permet de reconstituer le développement des populations depuis 1959. En ce qui concerne les facteurs du milicu forestier, les liaisons entre l'importance des populations d'une part, l'exposition, la densité des peuplements et l'essence hôte d'autre part sont confirmées. La généralité de l'incidence de ces facteurs est démontrée, tandis que l'eftet d'autres facteurs telle l'altitude dépend plus des conditions propres à chaque massif forestier. Deux périodes distinctes sont mises en évidence dans l'évolution des populations depuis 1970. Il leur correspond des répartitions différentes des populations dans la région étudiće. Les résultats confirment l'importance de caractéristiques comportementales de l'insecte dans la dynamique de ses populations et suggèrent l'incidence de facteurs dépendant de la densité des populations.
\end{abstract}

\section{Introduction}

La mesure des populations de processionnaire du pin (Thaumetopoea pityocampa Schiff.) par dénombrements des nids a été mise au point dans la vallée du Niolo en Corse (Dusaussoy \& Geri, 1970; Geri, 1971, 1980 a et b, 1983). 
Grâce à des recensements étayés par des observations biologiques et démographiques, les principales caractéristiques de la dynamique des populations de cet insecte ont pu être appréhendées dans les forêts de la montagne corse; des relations ont été mises en évidence entre les populations de processionnaires et la structure des peuplements de ces forêts très anciennes soumises aux seules interventions sylvicoles.

Ces techniques de dénombrement ont été adoptées en 1969 par les chercheurs de la station de Zoologie forestière de I'I.N.R.A. d'Avignon pour conforter les études biologiques des populations du Mont-Ventoux (Vaucluse) et interpréter les modifications de ces populations à la suite d'opérations de lutte. Nous avons mis au point le dispositif de sondage et analysé les données recueillies dans cet habitat beaucoup plus artificialisé que les forêts corses tant par l'implantation récente des boisements que par les interventions successives réalisées contre la processionnaire.

Cette analyse est présentée ici ; elle porte sur :

- les mesures des effectifs du massif forestier;

- les relations entre les populations et leur environnement physique et forestier ;

- les conséquences des traitements effectués;

- la contribution des mesures de population à la confirmation des phénomènes éthologiques et à la connaissance de la dynamique des populations.

L'apport de l'étude quantitative sur ce dernier point ne pourra cependant être totalement apprécié qu’en fonction des résultats des études biologiques.

\section{Description sommaire du massif}

\subsection{Caractéristiques des boisements}

Le Ventoux est une montagne calcaire qui présente de sa base $(400 \mathrm{~m})$ au sommet $(1909 \mathrm{~m})$ tous les étages de végétation, du méditerranéen au sub-alpin. Son orientation générale est Est-Ouest avec un versant nord et un versant sud bien marqués.

La forêt, anciennement détruite par l'homme, a fait l'objet de reboisements importants à partir de 1861 . Les essences utilisées ont été, par altitudes croissantes, le pin d'Alep, Pinus halepensis Mill., le pin maritime, $P$. pinaster Ait., le pin noir d'Autriche, $P$. nigra Arn. var. austriaca, le cèdre, Cedrus atlantica Manetti, le pin sylvestre, $P$. sylvestris L., ct le pin à crochets, $P$. uncinata Ramond.

Seules les extrémités est et oucst du massif sont sévèrement infestées, la face nord est généralement indemne et les attaques sont faibles dans la forêt de Bedoin sur le versant sud (Grison, Vago, Maury, 1959).

Le secteur étudié correspond à l'extrémité occidentale du massif (forêt domaniale de Malaucène et parties basses de la forêt de Beaumont). Il a un versant sud et un versant nord bien marqués. Les populations de processionnaire n'y dépassent guère $1000 \mathrm{~m}$ d'altitude. 
Les peuplements de ce secteur sont essentiellement composés de pins noirs d'Autriche en mélange avec des pins sylvestres et des cèdres (5 p. 100), de chênes pubescents et de chênes verts $(10 \mathrm{p}$. 100), et de rares pins d'Alep. Ils s'étendent sur environ 500 ha de 500 à $900 \mathrm{~m}$, altitude très inférieure à celle des peuplements de la montagne corse attaqués par la processionnaire $(800$ à $1500 \mathrm{~m})$. Les pins, provenant en majorité du boisement initial, y sont de taille plus modeste $(10 \mathrm{~m}$ en moyenne au lieu de 30 à $40 \mathrm{~m}$ ). Ils constituent des futaies régulières de 90 ans, plus ou moins clairiérées suivant la nature du terrain. Dans les sols profonds (fonds de combes) poussent des futaies denses à croissance rapide, tandis qu'aux sols superficiels où la roche mère affleure correspondent des boiscments clairs à végétation lente. La régénération, souvent partielle, est complétée par des reboisements artificiels.

Les beaux peuplements de pins noirs, purs ou mélangés à quelques cèdres et pins sylvestres, sont situés sur le versant nord, tandis que les boisements clairiérés sur sol peu profond où les pins noirs voisinent avec des cèdres, des pins sylvestres ou des chênes, sont plus fréquents sur le versant sud.

\subsection{Climat}

Le climat da Ventoux est de type méditerranéen avec des pluies abondantes au printemps et à l'automne et un été sec. Comme celui du Niolo (Pinguet \& Samie, 1980), il a fait l'objet d'une étude détaillée du Service technique d'Etudes des Facteurs climatiques de l'I.N.R.A. (Antonioletti \& Baculat, 1980).

Ces études mettent en évidence, dans les deux massifs forestiers, une décroissance nette des températures avec l'altitude et des écarts de température importants entre la face nord, plus froide, et la face sud.

Dans le secteur du Ventoux étudié (c'est-à-dire à moins de $900 \mathrm{~m}$ ), les températures, du fait de la différence altitudinale, sont régulièrement plus élevées que dans les peuplements du Niolo. Mais elles s'abaissent rapidement avec l'altitude au-dessus de $1000 \mathrm{~m}$ au Ventoux tandis que le nombre de jours de gelées augmente régulièrement. Dans le Niolo, du fait des conditions particulières de cette vallée (incidence des expositions, ouverture vers le golfe de Porto) et de sa plus faible latitude, cette décroissance est plus lente et très réduite pour les minima. Il en résulte un climat beatcoup plus froid en altitude au Ventoux.

\subsection{Caractéristiques des populations de processionnaires}

Le cycle évolutif de la processionnaire est influencé par ces différences climatiques.

Dans les deux massifs, la ponte a lieu en juillet et au début août ế les chenilles, après avoir tissé des nids épais, passent généralement l'hiver au quatrième stade. Mais elles terminent leur développement et partent en procession essentiellement en marsavril dans le site plus chaud du Ventoux, seulement en mai-juin dans la montagne corse. 


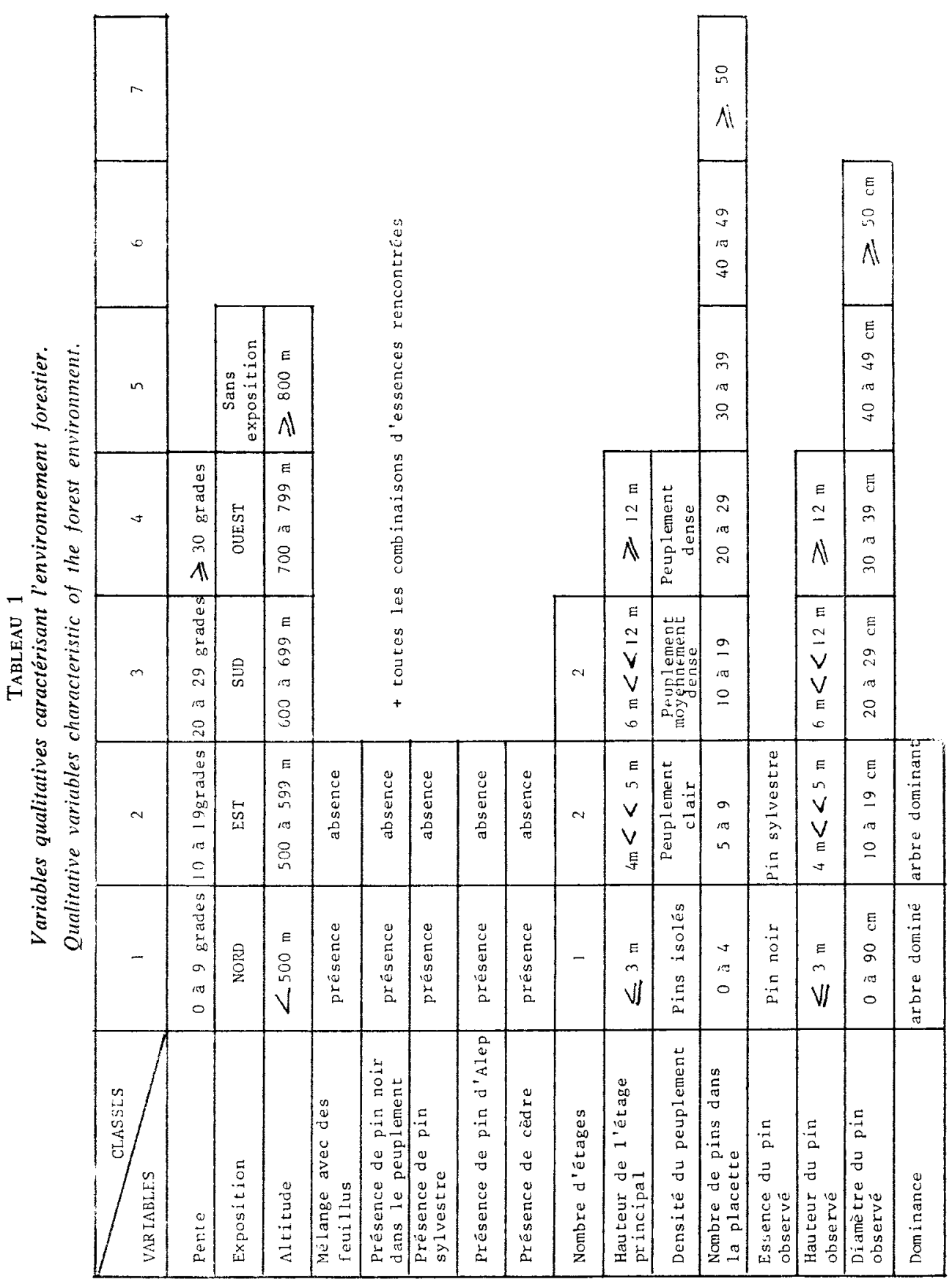


Dans ces conditions, les processionnaires de Corse ne peuvent achever leur cycle en un an et les chrysalides subissent régulièrement une diapause d'un an au moins (Demolin, 1969 a). Il s'ensuit un rythme bisannuel d'apparition des populations. Par contre, le climat relativement clément en altitude leur permet de se développer jusqu'à la limite altitudinale des peuplements.

An Ventoux, au-dessous de $1000 \mathrm{~m}$, le développement plus rapide de l'insecte permet un cycle annuel et la diapause n'intervient que de façon irrégulière, surtout en altitude et pour les populations tardives. Au-dessus de $1000 \mathrm{~m}$, les conditions climatiques deviennent limitantes, d'autant plus que les boisements de pins noirs sont alors fréquemment en exposition nord.

\section{Situation de l'étude}

dans l'historique des populations de processionnaire du Mont-Ventoux

Peu de temps après leur mise en place, les boisements du Ventoux ont été attaqués par la processionnaire. Des essais successifs de traitement ont été réalisés (GRISON et al., 1959) : injections de pétrole dans les nids, ramassage des nids jusqu'en 1925, ceinturage des troncs à la glu (1949), applications localisées d'insecticides de contact (1950), premières tentatives de lutte avec Bacillus thuringiensis et avec la virose cycloplastique en 1956 et 1957.

Les polyèdres de ce virus ont été répandus par hélicoptère sur une grande partie du secteur étudié ( $320 \mathrm{ha}$ ) en 1958 , puis sur sa totalité en 1959. Des contrôles biologiques confirmèrent la mortalité des chenilles et la présence de la maladie dans les zones traitées (Grison et al., 1959; Grison, 1969). Cette chute de population ne put cependant être attribuée avec certitude aux traitements, car des régressions furent également constatées dans les régions voisines. De plus les populations des zones traitées et témoins ne firent pas l'objet de mesures précises avant et après l'intervention.

Toutefois, grâce à l'ingénieur principal MaURY, à partir de 1959, les agents de l'O.N.F. ont dénombré tous les ans les nids dans trois placettes d'une soixantaine de pins situées dans laxe du massif traité, le long de la route conduisant de Malaucène au sommet.

Ces interventions furent suivies d'une longue période durant laquelle ne furent observées que de très faibles populations. L'infestation reprit en 1965, devint tr⿳े̀s grave en 1967 et régressa ensuite.

En 1969 fut mis en place le dispositif de recensement des populations présenté ici. Il a permis de suivre l'évolution naturelle des populations de 1969 à 1978. En 1979, une zone de plus de 120 ha (zone 2) a été traitée au diflubenzuron (Dimilin), insecticide inhibiteur de mue préalablement testé sur la processionnaire (DEMoLiN \& Millet, 1981). Le dispositif a alors servi à apprécier limpact de ce traitement. 


\section{Méthodes}

\subsection{Collecte des données}

L'estimation des populations est obtenue par un plan d'échantillonnage au hasard stratifié. La forêt a été découpée en 6 zones, dont 3 se succèdent en altitude sur le versant sud ( $n^{\prime \prime \prime} 1,2$ et 6 ) et 3 sur le versant nord $\left(n^{n s} 3,4\right.$ et 5). Chaque zone a été divisée en 3 sous-zones suivant la densité des peuplements. Celles-ci sont numérotées de 1 à 3 , pour la zone 1 , de 16 à 18 , pour la zone 6 . Dans chaque zone les numéros sont attribués par ordre croissant, aux peuplements clairs, moyennement denses puis denses.

Les points d'observation ont été tirés au hasard dans chaque sous-zone, sur carte, à raison d’un pin pour 1,5 ha pour les cycles 1969-1970 et 1970-1971. Par la suite, à la différence du Niolo, ce dernier tirage a été conservé et les mêmes pins sont observés d'année en année.

Chaque point est repéré sur le terrain à la boussole et par ses distances à des repères fixes (bornes kilométriques, constructions, intersections de chemins). Une placette de $400 \mathrm{~m}^{2}$ est délimitće autour de ce point et le pin le plus proche est marqué.

Chaque année, les nids, tissés avant lhiver, sont dénombrés sur les pins marqués au printemps. L'année de l'enquête correspond donc à la deuxième année du cycle biologique. 3 indices d'infestation sont également notés, suivant le code utilisé en Corse, après quelques modifications apportées par les chercheurs d'Avignon :

Au niveau du pin (I pin) :

0 ( 0 nid), 0,5 ( 1 nid, dégâts faibles), 1 ( 1 nid, dégâts nets), $1,5(\geqslant 1$ nid, défeuillaison du pin $<1 / 4$ ), 2 (défeuillaison $=1 / 4$ ), 2,5 (défeuillaison : $1 / 4$ à $1 / 2$ ), 3 (défeuillaison $=1 / 2), 3,5$ (défeuillaison : $1 / 2$ à $3 / 4), 4$ (défeuillaison $=3 / 4), 4,5$ (défeuillaison $>3 / 4$ ), 5 (défeuillaison totale).

Au niveau de la placette (IF) ou de son proche environnement (IP) :

0 (0 nid), 0,5 (1 nid, dégâts faibles), 1 (1 nid, dégâts nets), 1,5 (plusieurs nids, dégâts faibles), 2 (plusieurs nids, dégâts nets), 2,5 (1 nid par pin en moycnne, dégâts faibles), 3 (1 nid par pin en moyenne, dégâts nets), 3,5 $\geqslant 1$ nid par pin), 4 (2 nids par pin en moyenne), 4,5 ( $>2$ nids par pin en moyenne), 5 ( $>2$ nids par pin, dégâts importants).

Dans les placettes, un certain nombre d'observations ont été faites en 1970, puis en 1971 (tirage définitif) sur les peuplements :

- altitude, pente (en grades) et exposition,

- nombre de pins dans la placette,

- nombre d'étages (ensemble d'arbres de taille et d'âges voisins), et essences présentes,

- densité du peuplement (notée de 1 à 5 pour des densités croissantes, de l'arbre isolé au peuplement très épais), 
- hauteur moyenne de l'étage principal (étage présentant la plus grande masse foliaire), à vuc, en mètres,

- essence, hauteur en mètres et circonférences, en centimètres, du pin marqué.

\subsection{Analyse des données}

\subsection{Estimation des effectifs et de leurs fluctuations}

Le nombre de nids présents est estimé à chaque cycle dans chaque sous-zone par $\mathrm{T}=\mathrm{P} \overline{\mathrm{x}}$,

$\mathbf{P}$ et $\overline{\mathbf{x}}$ ćtant le nombre total de pins et le nombre moyen de nids par pin de la sous-zone.

$P$ est lui-même estimé par $P=\frac{1}{f} \Sigma p_{i}$,

où $\mathrm{p}_{\mathrm{i}}$ est le nombre de pins de chaque placette et $\mathrm{f}$ la fraction de sondage

$$
\left(\mathrm{f}=\frac{400 \mathrm{~m}^{2}}{1500 \mathrm{~m}^{2}}, \frac{1}{\mathrm{f}}=37,5\right)
$$

Dans cette étude on n'a pas tenu compte des espèces de pins, les pins sylvestres n'étant représentés que par une quarantaine d'individus et les deux espèces présentes étant très attaquées, même si les pins sylvestres ont dans l'ensemble des infestations un peu moins fortes.

L'estimation de la variance de $\mathrm{T}$ est approximativement :

$$
\operatorname{Var} \mathbf{T}=\operatorname{Var} P \overline{\mathrm{x}}=\mathrm{P}^{2} \operatorname{Var} \overline{\mathrm{x}}+\overline{\mathrm{x}}^{2} \operatorname{Var} \mathrm{P}+2 \overline{\mathrm{x}} \mathbf{P} \operatorname{cov}(\overline{\mathrm{x}} \mathrm{P})
$$

L'échantillonnage étant statifié, les populations des zones et de l'ensemble du massif et leurs variances sont obtenues par simple sommation de ces estimations. Cette sommation peut également être effectuée pour les différents types de peuplement (clairs, moyennement denses et denses), distingués pour constituer les souszones.

Nous avons étudié comment le terme $2 \bar{x} \mathbf{P} \operatorname{cov}(\bar{x} P)$, négligé dans les études précédentes (GERI, 1971, $1980 \mathrm{a}$ et b, 1983), modifiait le coefficient de variation de ces estimations.

Les fluctuations de populations (D) entre deux années consécutives $n$ et $n+1$ sont calculées par :

d'où :

$$
\mathrm{D}=\mathrm{T}_{\mathrm{n}}-\mathrm{T}_{\mathrm{n}-1}
$$

$$
\operatorname{Var} D=\operatorname{Var} T_{n}+\operatorname{Var} T_{n-1}-2 \operatorname{cov}\left(T_{n}, T_{n-1}\right)
$$

Le terme de covariance peut être calculé puisque le dispositif de sondage sélectionne les mêmes pins à chaque cycle; il s'écrit :

$$
\begin{gathered}
\operatorname{Cov}\left(T_{n}, T_{n-1}\right)=P^{2} \operatorname{cov}\left(\bar{x}_{n}, \bar{x}_{n-1}\right)+\bar{x}_{n} \vec{x}_{n-1} \operatorname{Var} P+P \bar{x}_{n-1} \operatorname{cov}\left(P, \bar{x}_{n}\right), \\
+P \bar{x}_{n} \operatorname{cov}\left(P, \bar{x}_{n-1}\right)
\end{gathered}
$$


L'estimation de Var D ainsi obtenue est comparée à :

$$
\operatorname{Var} \mathrm{D}=\operatorname{Var} \mathrm{T}_{n}+\operatorname{Var} \mathrm{T}_{n-1}
$$

Estimation qui conviendrait si les pins étaient tirés au sort à chaque cycle.

\subsection{Etude des relations entre les populations et leur milieu}

\subsection{Corrélations et tableaux de contingence}

Les corrélations simples entre les mesures de population au nivcau des pins, entre ces mesures et les variables du milieu forestier, et entre ces variables du milieu entre elles, ont été calculées une première fois de 1971 à 1977, sous-zone par sous-zone, puis pour l'ensemble du massif, en distinguant les corrélations inter (entre souszones) et intra (à l'intérieur des sous-zones) (GERI, 1980 a); puis la matrice générale de corrélations a été établie de 1971 à 1979.

Pour les mesures de population on a pris en compte les nombres de nids et les indices d'infestation des différents cycles, le nombre de nids recensés sur l'ensemble des années (variable «total») et le nombre d'années sans attaques (variables "années») ainsi que les variations de populations sous leur forme absolue (nombre de nids - nombre de nids du cycle précédent) et relative (nombre de nids $+1 /$ nombre de nids du cycle précédent +1 ).

Une étude préalable ayant montré que la distribution du nombre de nids par pin suit une loi binomiale négative, on a également introduit le logarithme de cette variable pour égaliser les variances, en lui ajoutant 1 pour éviter le logarithme de nombres nuls.

Pour les factcurs du milicu l'exposition est traduite par un indice 1,0 ( 1 sud, 0 nord) ou par son cosinus et son sinus, la présence de feuillus et des différents conifères ainsi que la situation des pins (dominants ou dominés) sont également appréciées par une notation 0 et 1 .

Dans chaque zone la répartition des facteurs du milieu, transformés en variables qualitatives, a été étudiée plus précisément par un tableau de contingence (tabl. 2).

Des analyses statistiques plus élaborées ont été développées dans deux directions :

- recherche des liaisons entre les effectifs des populations et leur environnement forestier ;

- incidence des facteurs du milieu (populations antérieures, composantes du milieu forestier, interventions humaines) sur les variations de ces effectifs.

\subsection{Etude des liaisons entre les niveaux de populations et les caractéristiques du milieu forestier}

Ces liaisons ont été testées par l'analyse de variance multivariable non orthogonale. Cette analyse, décrite notamment par Bachacou, Masson, Millier (1981), consiste à estimer un modèle rendant compte de la population de chaque pin en fonction des facteurs du peuplement transformés en variables qualitatives à plusieurs classes (tabl. 1). 


\section{(⿸丆口}

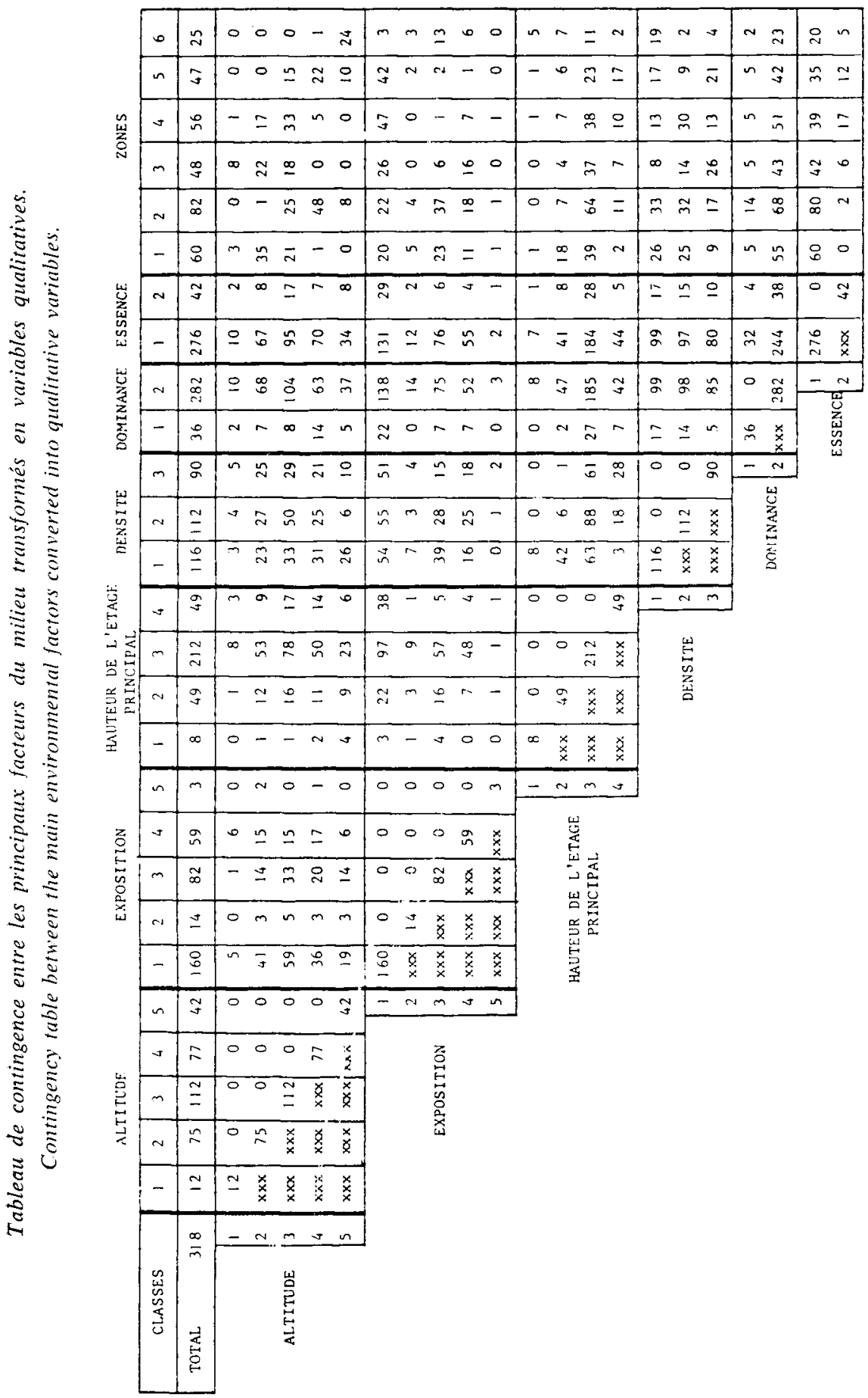


On propose un modèle additif décomposant pour chaque cycle l'action de ces facteurs et de leurs interactions d'ordre 2 sur la variable $Z=\log$ (nombre de nids +1 ), sur les indices d'infestation et, pour l'ensemble des cycles, sur les variables «TOTAL » et «ANNEES».

11 s'écrit : où :$$
Z=\mu+a_{i}+b_{j}+c_{k}+d_{1}+e_{m}+f_{n}+g_{w}+(a b)_{i j}+\ldots+(f g)_{n_{0}}+\xi
$$

14 représente la moyenne générale,

$\mathrm{a}_{\mathrm{i}}, \ldots \mathrm{g}_{0}$ les effets dus à l'appartenance du pin aux différentes classes d'altitude, d'exposition, de densité, de hauteur des peuplements, de nombre d'étages et d'essence,

$(\mathrm{ab})_{\mathrm{ij}}, \ldots(\mathrm{fg})_{\mathrm{no}}$ les effets des interactions entre ces facteurs,

$\xi$ l'élément aléatoire.

Un modèle peu différent :

$\mathrm{Z}=\mu+\mathrm{a}_{\mathrm{i}}+\mathrm{b}_{\mathrm{j}}+\mathrm{c}_{\mathrm{k}}+\mathrm{d}_{\mathrm{i}}+\mathrm{e}_{\mathrm{m}}+\mathrm{f}_{\mathrm{u}}+\mathrm{g}_{\mathrm{r}}+\mathrm{h}_{\%}+(\mathrm{ab})_{\mathrm{ij}}+\ldots+(g h)_{0 \%}+\xi$

teste l'incidence qu'ont encore ces différents facteurs du milieu après avoir testé l'effet $h_{\%}$ de leur regroupement dans des unités territoriales présentant des caractères géographiques déterminés (zones).

L'action de chaque facteur est testée par un test $\mathrm{F}$ de Fisher, indépendamment des autres caractéristiques du milieu ramenées à leur valeur moyenne. La transformation en facteurs qualitatifs correspond à une perte dinformations mais assure une certaine robustesse du modèle compte tenu de la méconnaissance du type de liaison entre l'infestation et les facteurs du milieu.

La représentation graphique de la moyenne générale $\mu$ et des modifications dues à l'appartenance des pins aux différentes classes de chaque facteur $\left(\mathfrak{\imath}+\Lambda_{\text {̣ }}\right.$ ) illustre ces relations (figure 3 ).

\subsection{Analyse multivatute des fluctuations de population}

\section{- Développement du modèle précédent}

Ces analyses tiennent compte des infestations des années antéricures, ou de modifications artificielles qu'elles ont subies (traitement), pour expliquer les liaisons entre les populations et leur milieu. Réciproquement, elles cherchent à apprécier l'incidence de ce dernier sur les fluctuations de populations observées.

Grâce à léchantillon de pins permanent, l'analyse de variance non orthogonale a pu être utilisée à cet effet sur les données du Ventoux. En appliquant le modèle (1) aux populations de deux annécs consécutives $n$ et $n+1$ on obtient, en appelant $N$ le nombre de nids :

$$
\begin{aligned}
Z_{n}-Z_{12-1}=\log \left(N_{n}\right. & +1)-\log \left(N_{n-1}+1\right)=\log \frac{N_{n}+1}{N_{n-1}+1} \\
& =! \iota_{11}-l_{n 1}+C
\end{aligned}
$$

où $\mathrm{C}$ est l'ensemble des termes rendant compte de laction des facteurs du milieu sur l'accroissement relatif des populations d'année en année; l'effet dû à chaque classe de facteur étant égal à la différence des effets constatés au cours des deux années. 
Le modèle (1) a été également développé en prenant en compte la covariance entre les populations de deux années successives et en testant les facteurs du milieu sur le résidu.

et :

$$
Z_{11}=l_{n}+\lambda\left(Z_{n-1}-u_{n-1}\right)+C^{\prime}
$$

$$
Z_{\mathrm{n}}-\mathrm{Z}_{\mathrm{a} \cdots-1}=\mathrm{l}_{\mathrm{n}}-\lambda \mathrm{l}_{\mathrm{n}-1-1}+(\lambda-1) \mathrm{Z}_{\mathrm{n}},+\mathrm{C}^{\prime}
$$

où $\lambda$ est le coefficient de régression de $Z_{n}$ avec $Z_{n-1}$,

et $C^{\prime}$ l'ensemble des termes décrivant l'action des facteurs du milieu après avoir tenu compte de la population de l'année $\mathrm{n}$.

En revenant aux variables $\mathrm{N}$ les deux modèles s'écrivent :

$$
\mathrm{N}_{n}=-1+\mathrm{A}\left(\mathrm{N}_{n \ldots 1}+1\right) \mathrm{e}^{\mathrm{c}}
$$

et :

$$
\mathrm{N}_{n}=-1+\mathrm{A}\left(\mathrm{N}_{n \ldots 1}+1\right)^{\lambda} \mathrm{e}^{\mathrm{x}}
$$

L'incidence des facteurs de l'environnement est multiplicative avec les niveaux de population. Ce mode est vraisemblable; ainsi un peuplement dense n'est pas peuplé de la même façon par de fortes ou de faibles populations. Mais si le premier modèle ne fait intervenir qu'une relation de proportionnalité entre les populations consécutives (GERI, $1980 \mathrm{a}$ et b, $1983 \mathrm{a}$ ), le second est basé sur une fonction plus complexe, permettant en principe de faire apparaître des effets négatifs de la densité des populations lorsque $\lambda$ est inférieur à 1 (Morris, 1963).

Ces effets négatifs présumés des fortes populations, notamment à cause de la rareté de la nourriture et de sa modification qualitative, ont également fait l'objet d'une approche simple, plus proche de la réalité biologique, à partir du premier modèle. On a considéré que les phénomènes de jeûnes interviennent avec certitude si l'indice d'infestation du pin est supérieur à 3,5 ; le cycle biologique ne pouvant alors être accompli sans défeuillaison totale des arbres.

On a créé l'indice «SURP»=1 si I pin $>3,5,0$ sinon, et testé, en considérant que ces phénomènes se répercutent surtout sur le nombre de nids recensés l'année suivante, les modèles :

$$
\begin{gathered}
Z_{n}=\eta+\Delta_{i \ell} \ll S U R P »_{n-1}+C \\
Z_{n}-Z_{n-1}=\eta+\Delta_{i \ell} \ll \operatorname{SURP} »_{n-1}+C
\end{gathered}
$$

o» $\Delta$ lı «SURP $\gg$ représente les effets de cet indice en sus de ceux des facteurs du milieu, C, dans les modèles (1) et (3).

De la même façon, l'incidence du traitement de 1979 a été appréciée par l'indice *TRAIT $»=1$ si le pin est dans la zone 2,0 sinon, et en testant les modèles :

$$
\begin{aligned}
& Z_{\mathrm{u}}=\imath+\Delta_{\mathfrak{\imath}} \ll \text { TRAIT } »+\mathrm{C}
\end{aligned}
$$

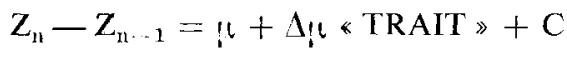

$$
\begin{aligned}
& \text { I pin }=\imath+\Delta ! t * \text { TRAIT } »+C
\end{aligned}
$$

\section{- Analyses factorielles}

L'analyse en composantes principales (cf. CAILliEz et al., 1971, 1976) a été utilisée pour rendre compte simultanément de l'évolution de l'infestation, du compor- 
tement des populations des différentes zones et de l'incidence des facteurs du milieu antérieurement au traitement.

Elle a porté sur les variables Z de 1971 à 1979 et sur les caractéristiques de l'environnement forestier notées en 1971.

Les distances entre arbres ont été calculées avec la métrique des covariances (distances euclidiennes simples) puis avec la métrique des corrélations (distances euclidiennes pondérées par la variance) pour accorder une importance égale aux années de faibles et de fortes infestations.

Seules les mesures de populations (variables de même nature ayant la même unité (le mesure) ont servi au calcul des axes principaux, les caractéristiques du milieu étant seulement prises en compte comme variables supplémentaires et considérant leurs corrélations avec ces axes. Les calculs ont été réalisés sur les données relatives au pin noir, puis sur l'ensemble des clonnées.

La répartition différente des populations dans le massif au cours de leur évolution, mise en évidence par cette analyse, a ensuite été approfondie par l'analyse discriminante entre sous-zones (cf. Bachacou et al., 1981, CaIlliez et al., 1971, 1976). Dans cette analyse, comme dans la précédente, le nuage multidimensionnel des points est réduit par rapport à un nombre limité d’axes (axes canoniques). Ces axes minimisent les distances entre les individus d'une même sous-zone et distinguent au maximum les sous-zones cntre elles, les distances étant calculées suivant une métrique statistique (distance de Mahalonobis).

\section{Recensement des populations}

\subsection{Résultats et erreurs d'échantillonnage}

Les estimations du nombre moyen de nids par pin $(\bar{x})$, du nombre total de nids $(T)$ et de ses fluctuations d'un cycle à l'autre $\left(T_{n}-T_{n-1}\right)$, pour les zones, les types de peuplement et l'ensemble du massif, sont présentées avec leurs coefficients de variation sur les tableaux 3,4 et 5 . Ceux-ci sont calculés comparativement pour $\mathrm{T}$, en négligeant le terme de covariance et en le conservant, et pour $T_{n}-T_{n-1}$, en considérant les notations comme indépendantes à chaque cycle puis en tenant compte du fait qu'elles sont réalisées sur les mêmes pins.

Si l'on considère comme erreur le demi-intervalle de confiance en valeur relative (cocfficient de variation $\times \mathrm{t}$ de Student), l'erreur sur les évaluations de $\mathrm{T}$ est 18 p. 100 les années de fortes infestations mais peut atteindre 50 p. 100 sur les faibles populations pour l'ensemble du massif. Elle se situe entre 20 et 150 p. 100 pour les types de peuplements, entre 20 et 200 p. 100 pour les zones; pour les estimations relatives à toutes les sous-zones dont certaines n'ont que quelques points d'observations qui n'ont pu être rapportées sur les tableaux cette erreur est comprise en 30 et 300 p. 100 . La prise en considération du terme de covariance la réduit d'environ 10 p. 100.

Les erreurs sur les fluctuations de population sont plus élevées; elles restent cependant dans des limites voisines pour les zones et pour le massif. Le gain de pré- 
cision dû à la mesure sur les mêmes pins est appréciable (plus de 80 p. 100) pour des populations successives très corrélées, correspondant généralement à des infestations importantes. Ces erreurs, bien que relativement importantes, sont généralement inférieures aux fluctuations de populations du massif et des différentes zones (fig. 1). Malgré le nombre limité de pins observés (318), elles permettent, sauf dans les souszones d'effectif très réduit, de suivre l'évolution des populations. Comme dans les études précédentes (GERI, 1971, 1980 a et b, 1983), la stratification se traduit par une réduction importante de l'erreur.

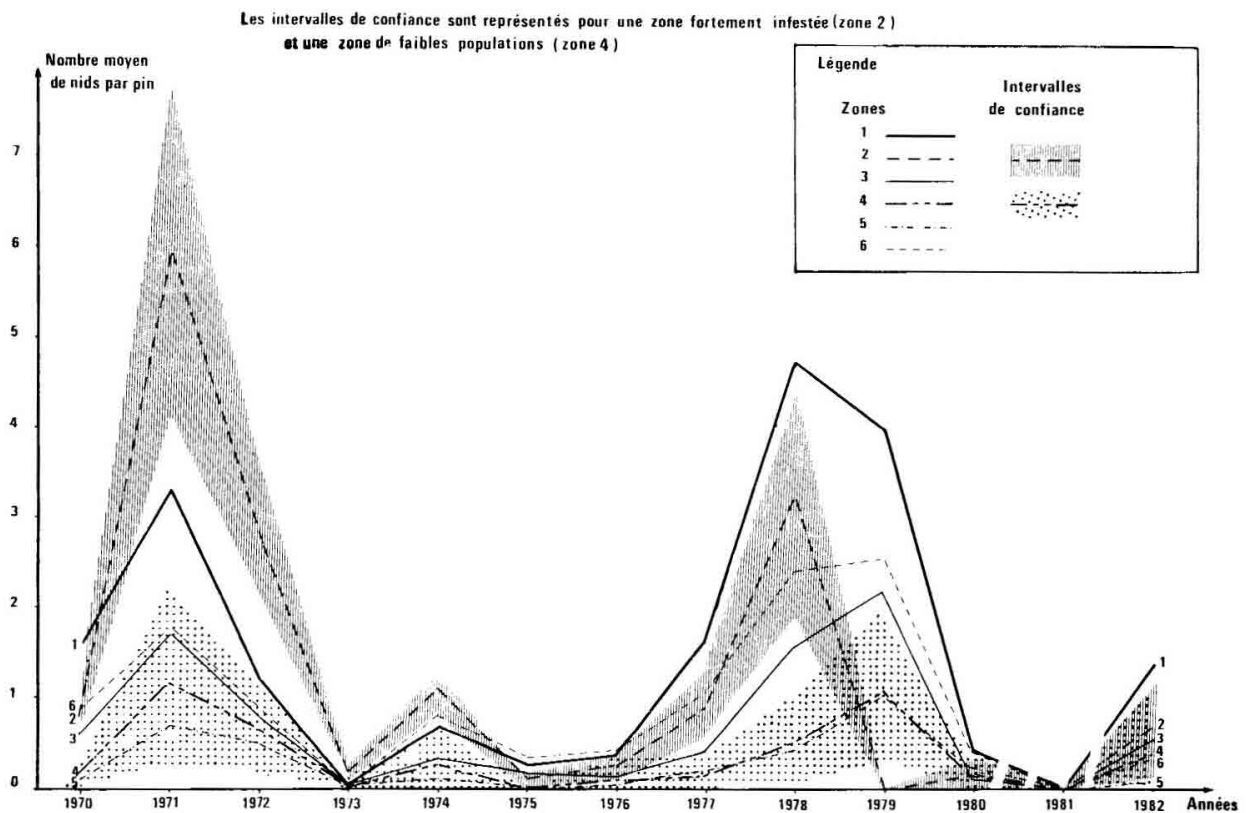

FIG. 1

Nombres moyens de nids par pin par zone de 1970 à 1982.

Mean number of nests per pine in each zone from 1970 to 1982.

\subsection{Répartition des populations}

Il apparaît sur le tableau 3 et sur la figure 1 que les zones les plus infestées le demeurent d'année en année. Cette répartition des populations dépend du milieu : le nombre moyen de nids par pin est plus élevé sur la face sud que sur la face nord et dans les peuplements clairs que dans les peuplements moyennement denses ou denses. Ces derniers ont cependant, en raison de leur superficie et de leurs effectifs de pins, des populations importantes durant les années de fortes infestations. 


\section{TABLEAU 3}

Estimations du nombre moyen de nids par pin $(x)$ par type de peuplement et pour l'ensemble du massif, de 1970 à 1982. puis de son coefficient de variation $(\sigma \bar{x} / x) *$ en p. 100, par zone,

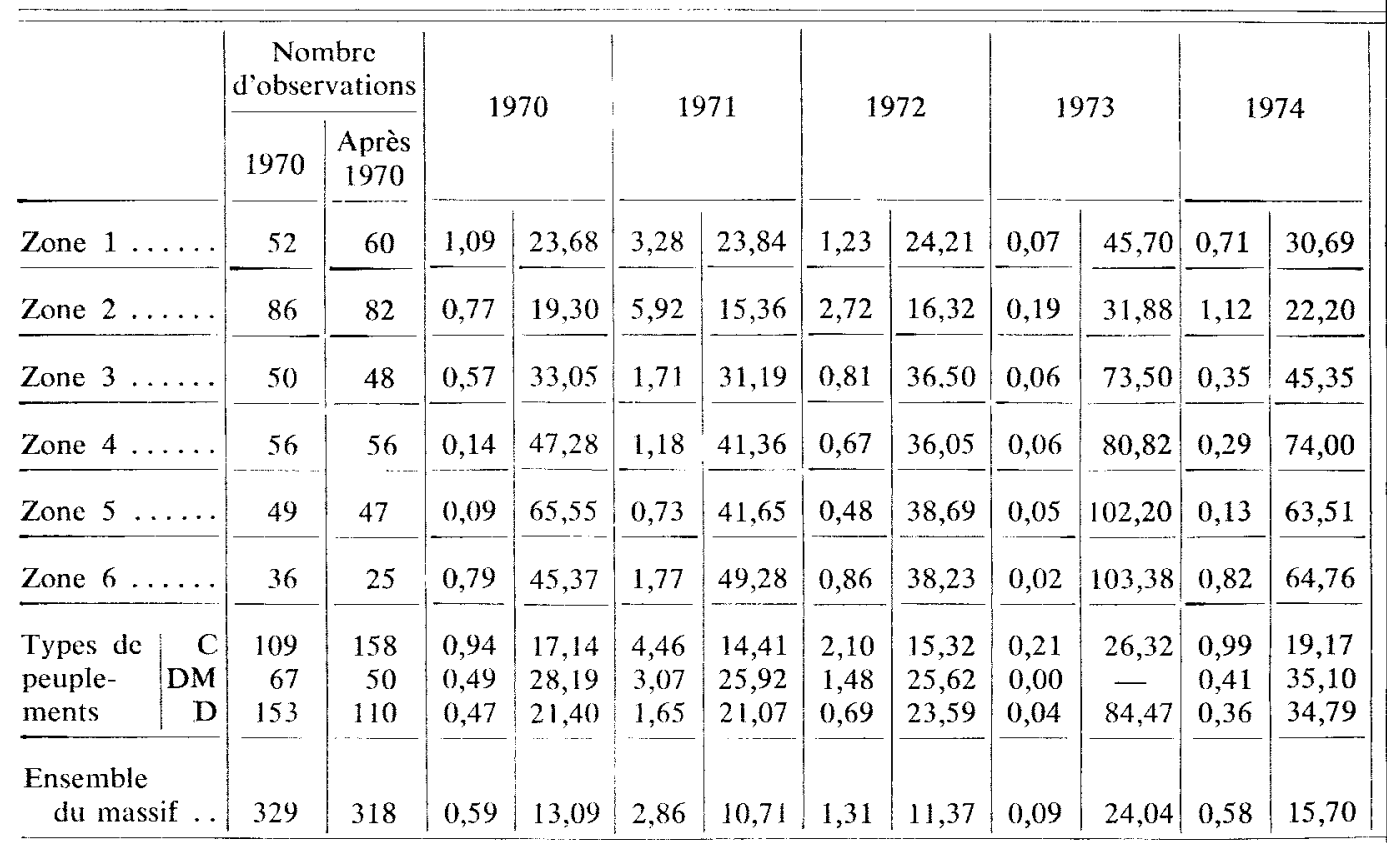

- $\overline{\mathbf{x}}$ est estimé par le rapport entre le nombre total de nids, $T$, et le nombre total de pins $\mathbf{P}$; cefte estimation, qui tient compte de la variation de densité dans le peuplement, a été préférée à la moyenne brute du nombre de nids par pin.

\subsection{Evolution des populations de 1970 à 1982}

Le premier recensement (cycle 1969-1970), effectué après une période de forts dégâts puis de régression, montre un effectif très faible pour toute laire d'étude. Au cycle suivant, cette population, est multipliée environ 5 fois dans toutes les zones et dans tous les types de peuplements, probablement à la suite d'émergence d'individus diapausants (Demolin, 1974). Elle régresse à nouveau durant les cycles 1971-1972, puis 1972-1973 où les effectifs des sous-zones sont réduits en moyenne all $20^{\circ}$ de leur niveau de 1971.

Une nouvelle recrudescence de linfestation, peut-être liée à des émergences d'individus diapausants, est enregistrée en 1973-1974, puis la population revient en 19741975 à un minimum identique à celui de 1973. Ces phénomènes sont relativement homogènes dans l'ensemble du massif, toutefois les populations des peuplements denses paraissent proportionnellement moins réduites. La diminution est importante dans la zone 2, la plus peuplée depuis 1971, dont le nombre de nids par pin devient inférieur à celui des autres zones du versant sud et même à celui de la zone 4 sur le versant nord. 
Estimates of the average number of nests per pine tree $(x)$ in each stand type and for the whole area, from 1970 to 1982 , and estimated coefficient of variation $(\sigma \bar{x} / x)^{*}$ in p. 100 in each zone.

\begin{tabular}{|c|c|c|c|c|c|c|c|c|c|c|c|c|c|}
\hline \multicolumn{2}{|c|}{1975} & \multicolumn{2}{|c|}{1976} & \multicolumn{2}{|c|}{1977} & \multicolumn{2}{|c|}{1978} & \multicolumn{2}{|c|}{1979} & \multicolumn{2}{|c|}{1980} & \multicolumn{2}{|c|}{1982} \\
\hline 0,27 & 28,84 & 0,40 & 29,11 & 1,65 & 24,74 & 4,74 & 20,25 & 4,01 & 20,76 & 0,46 & 29,45 & 1,41 & 25,90 \\
\hline 0,11 & 42,03 & 0,27 & 28,54 & 0,95 & 21,20 & 3,30 & 19,96 & 0,00 & 一 & 0,16 & 46,39 & 0,74 & 40,99 \\
\hline 0,16 & 66,87 & 0,13 & 48,25 & 0,41 & 41,13 & 1,60 & 28,91 & 2,19 & 36,72 & 0,12 & 42,89 & 0,57 & 49,85 \\
\hline 0,00 & - & 0,10 & 101,03 & 0,15 & 59,41 & 0,52 & 46,50 & $1,1 !$ & 36,56 & 0,14 & 66,63 & 0,41 & 101,01 \\
\hline 0,01 & 105,21 & 0,05 & 61,43 & 0,22 & 43,28 & 0,45 & 44,69 & $1,11)$ & 37,49 & 0,22 & 44,62 & 0,08 & 51,92 \\
\hline 0,37 & 98,29 & 0,45 & 53,29 & 1,08 & 52,33 & 2,42 & 34,40 & 2,58 & 37,87 & 0,45 & 44,98 & 0,39 & 25,75 \\
\hline 0,16 & 25,01 & 0,48 & 20,06 & 1,57 & 17,24 & 4,45 & 15,34 & 2,68 & 18,72 & 0,36 & 25,38 & 1,63 & 24,52 \\
\hline 0,05 & 73,76 & 0,10 & 66,10 & 0,29 & 37,85 & 1,72 & 30,34 & 0,88 & 39,16 & 0,28 & 31,85 & 0,11 & 72,24 \\
\hline 0,12 & 48,63 & 0,05 & 58,60 & 0,27 & 28,22 & 0,95 & 21,93 & 1,16 & 31,88 & 0,09 & 40,76 & 0,19 & 40,11 \\
\hline 0,12 & 26,06 & 0,21 & 17,93 & 0,72 & 13,74 & 2,28 & 11,54 & 1,63 & 15,57 & 0,22 & $\mathrm{I} 8,1 \mathrm{I}$ & 0,67 & 20,89 \\
\hline & $\begin{array}{l}\mathrm{C} \\
\mathrm{MI} \\
\mathrm{D}\end{array}$ & $\begin{array}{l}: \mathrm{Pe} \\
: \mathrm{P}\end{array}$ & $\begin{array}{l}\text { lement } \\
\text { plement }\end{array}$ & $\begin{array}{l}\text { clair. } \\
\text { moye } \\
\text { dense }\end{array}$ & emer & Inense & & & & & & & \\
\hline
\end{tabular}

On observe ensuite une croissance progressive et continue des populations d'année en année jusqu'au cycle 1977-1978. Elle débute en 1975-1976, surtout dans les peuplements clairs alors que la population diminue encore dans les denses. Le nombre de nids par pin de la zone 2 qui atteint à nouveau des valeurs élevées, est cependant réduit par rapport aux autres zones et demeure inférieur à celui de la zone 1 .

Durant le cycle 1978-1979, la population de la zone 2 traitée est totalement détruite. Celle des autres zones continue à s'accroître, sauf dans les peuplements clairs de la zone 1, surpeuplés en 1978 (plus de 6 nids par pin).

Une régression générale des populations du massif est ensuite observée. Les populations de toutes les sous-zones sont réduites de plus de 7 fois en moyenne en 1979-1980. sauf dans la zone 2 où un début de repeuplement est noté. Elles disparaissent pratiquement durant le cycle 1980-1981. A cause du très faibie niveau des populations, l'enquête n'a pas été réalisée pour ce cycle.

La remontée, très rapide en 1981-1982 s'effectue préférentiellement dans les peuplements clairs, 10 fois plus peuplés en moyenne, et dans les zones sud. La zone 2 reste moins infestée que la 1 mais est à nouveau très attaquée. 


\section{TABleau 4}

Estimation des populations $(T)$ suivie de leurs coefficients de variations en p. 100. sans tenir compte du terme de covariance puis en en tenant compte,

par zone, par type de peuplement, et pour l'ensemble du massif de 1970 à 1982.

\begin{tabular}{|c|c|c|c|c|c|c|c|c|c|}
\hline \multirow{2}{*}{\multicolumn{2}{|c|}{$\overline{\text { Zone } 1 \ldots \ldots \ldots}$}} & \multicolumn{2}{|c|}{1970} & \multicolumn{3}{|c|}{1971} & \multicolumn{3}{|c|}{1972} \\
\hline & & 34976 & 23,66 & 138766 & 21,70 & 18,61 & 52083 & 22,11 & 18,59 \\
\hline \multicolumn{2}{|l|}{ Zone 2} & 50630 & 57,81 & 363046 & 13,24 & 11,60 & 166547 & 14,34 & 12,24 \\
\hline \multicolumn{2}{|c|}{ Zone $3 \ldots \ldots \ldots$} & 34312 & 33,04 & 97596 & 30,03 & 27,12 & 46381 & 35,52 & 32,41 \\
\hline \multicolumn{2}{|c|}{ Zone $4 \ldots \ldots \ldots$} & 6110 & 47,26 & 44824 & 40,63 & 39,50 & 25417 & 35,20 & 33,49 \\
\hline \multicolumn{2}{|c|}{ Zone $5 \ldots \ldots$} & 2742 & 65,48 & 24707 & 39,63 & 37,18 & 16265 & 36,51 & 32,31 \\
\hline \multicolumn{2}{|c|}{ Zone $6 \ldots \ldots \ldots$} & 6199 & 45,31 & 8362 & 46,75 & 46,07 & 4075 & 34,97 & 28,44 \\
\hline $\begin{array}{l}\text { Type de } \\
\text { peuplement }\end{array}$ & $\begin{array}{ll}\mathrm{C} & \ldots \\
\mathrm{D} & \ldots \\
\mathrm{MD}\end{array}$ & $\begin{array}{l}43928 \\
22477 \\
68562\end{array}$ & $\begin{array}{l}17,12 \\
18,23 \\
21,51\end{array}$ & $\begin{array}{l}362151 \\
124249 \\
190902\end{array}$ & $\begin{array}{l}12,73 \\
24,22 \\
20,22\end{array}$ & $\begin{array}{l}11,50 \\
20,79 \\
17,78\end{array}$ & $\begin{array}{r}170481 \\
60024 \\
80262\end{array}$ & $\begin{array}{l}13,76 \\
23,90 \\
22,83\end{array}$ & $\begin{array}{l}12,55 \\
18,11 \\
20,10\end{array}$ \\
\hline \multicolumn{2}{|c|}{ Ensemble du massif } & 134969 & 13,09 & 677302 & 9,93 & 8,80 & 310767 & 10,63 & 9,31 \\
\hline
\end{tabular}

TABLeau 4 (suite)

\begin{tabular}{|c|c|c|c|c|c|c|c|c|c|}
\hline \multirow[b]{2}{*}{ Zone $1 \ldots \ldots \ldots$} & \multicolumn{3}{|c|}{1973} & \multicolumn{3}{|c|}{1974} & \multicolumn{3}{|c|}{1975} \\
\hline & 2834 & 44,64 & 42,34 & 30153 & 29,07 & 25,08 & 11239 & 27,10 & 24,16 \\
\hline Zone 2 . & 11876 & 30,92 & 29,25 & 68545 & 20,78 & 18,05 & 6702 & 41,30 & 41,03 \\
\hline Zone $3 \ldots \ldots$ & 3321 & 73,011 & 68,03 & 20083 & 44,56 & 42,26 & 9045 & 66,34 & 63,82 \\
\hline Zone 4 & 2361 & 80.41 & 80,98 & 10997 & 73,59 & 72,01 & 0 & - & - \\
\hline Zone 5 & 1809 & 101,39 & 98,63 & 4331 & 62,20 & 57,96 & 309 & 104,32 & 104,9 \\
\hline Zone $6 \ldots \ldots \ldots$ & 106 & 101,88 & 112,26 & 3875 & 62,89 & 58,48 & 1737 & 97,06 & 87,22 \\
\hline \begin{tabular}{l|ll|} 
Type de & $\mathrm{C}$ & $\ldots$ \\
peuplement & $\mathrm{D}$ & $\ldots$ \\
$\mathrm{MD}$
\end{tabular} & $\begin{array}{r}17695 \\
0 \\
4613\end{array}$ & $\frac{25,44}{60,07}$ & $\begin{array}{l}23,92 \\
\overline{59,51}\end{array}$ & $\begin{array}{l}80187 \\
16518 \\
41280\end{array}$ & $\begin{array}{l}17,94 \\
33,86 \\
34,28\end{array}$ & $\begin{array}{l}16,46 \\
30,40 \\
30,36\end{array}$ & $\begin{array}{r}13290 \\
2116 \\
13627\end{array}$ & $\begin{array}{l}24,09 \\
73,16 \\
48,21\end{array}$ & $\begin{array}{l}21,48 \\
70,23 \\
46,57\end{array}$ \\
\hline Ensemble du massif & 22308 & 23,69 & 22,62 & 137985 & 15,18 & 13,68 & 29033 & 25,75 & 24,51 \\
\hline
\end{tabular}




\section{TABlenu 4 (stuite)}

Population estimates ( $T$ ) followed by their coefficients of variations in $p .100$ without considering the covariance term in the first table,

but considering it in the second table, in each zone,

each stand type and for the whole area from 1970 to 1982.

\begin{tabular}{|c|c|c|c|c|c|c|c|c|c|c|}
\hline \multirow{2}{*}{\multicolumn{2}{|c|}{ Zone $1 \ldots \ldots \ldots$}} & \multicolumn{3}{|c|}{1976} & \multicolumn{3}{|c|}{1977} & \multicolumn{3}{|c|}{1978} \\
\hline & & 16798 & 27,39 & 23,68 & 69884 & 22,70 & 18,44 & 200871 & 17,69 & 13,76 \\
\hline \multicolumn{2}{|l|}{ Zone 2} & 16447 & 27,46 & 26,76 & 58109 & 19,72 & 17,76 & 202050 & 18,38 & 16,20 \\
\hline \multicolumn{2}{|l|}{ Zone 3} & 553 & 47,50 & 46,07 & 23323 & 40,26 & 36,02 & 91381 & 27,66 & 24,13 \\
\hline \multicolumn{2}{|c|}{ Zone $4 \ldots \ldots \ldots$} & 3927 & 100,71 & 99,87 & 5860 & 54,81 & 56,20 & 19762 & 45,85 & 44,70 \\
\hline \multicolumn{2}{|l|}{ Zone 5} & 1856 & 60,07 & 59,95 & 7378 & 41,34 & 33,30 & 15028 & 42,82 & 41,2 \\
\hline \multicolumn{2}{|c|}{ Zone $6 \ldots \ldots \ldots$} & 2137 & 51,01 & 53,50 & 5100 & 49,88 & 56.16 & 11419 & 30,72 & 33,3 \\
\hline $\begin{array}{l}\text { Type de } \\
\text { peuplement }\end{array}$ & $\left|\begin{array}{ll}\mathrm{C} & \ldots \\
\mathrm{D} & \ldots \\
\mathrm{MD} & .\end{array}\right|$ & $\begin{array}{r}38650 \\
4368 \\
5701\end{array}$ & $\begin{array}{l}18,89 \\
65,44 \\
58,30\end{array}$ & $\begin{array}{l}17,71 \\
62,82 \\
55,67\end{array}$ & $\begin{array}{r}127058 \\
11827 \\
30679\end{array}$ & $\begin{array}{l}15,86 \\
36,71 \\
27,59\end{array}$ & $\begin{array}{l}13,56 \\
32,58 \\
24,46\end{array}$ & $\begin{array}{r}360790 \\
69681 \\
110040\end{array}$ & $\begin{array}{l}13,18 \\
28,90 \\
21,11\end{array}$ & $\begin{array}{l}11,68 \\
23,22 \\
18,57\end{array}$ \\
\hline \multicolumn{2}{|c|}{ Ensemble du massif } & 48719 & 17,48 & 16,47 & 169653 & 13,14 & 11,31 & 540510 & 10,82 & 9,16 \\
\hline
\end{tabular}

Tabliau 4 (suite)

\begin{tabular}{|c|c|c|c|c|c|c|c|c|c|c|}
\hline \multirow{2}{*}{\multicolumn{2}{|c|}{ Zone $1 \ldots \ldots \ldots$}} & \multicolumn{3}{|c|}{1979} & \multicolumn{3}{|c|}{1980} & \multicolumn{3}{|c|}{1982} \\
\hline & & 169800 & 18,27 & 15.61 & 19556 & 27,75 & 28,41 & 59672 & 23,96 & 19,58 \\
\hline \multicolumn{2}{|l|}{ Zone } & 0 & $\ldots$ & - & 9868 & 45,73 & 43,99 & 45628 & $+0,25$ & 38,8 \\
\hline \multicolumn{2}{|l|}{ Zone 3} & 125150 & 35,74 & 32,49 & 6889 & 42,05 & 42,24 & 32705 & 49,13 & 43,7 \\
\hline \multicolumn{2}{|l|}{ Zone 4} & 42315 & 35,73 & 35,42 & & 66,18 & 65,14 & 15706 & 100,71 & 99,8 \\
\hline \multicolumn{2}{|c|}{ Zone $5 \ldots \ldots$. } & 37165 & 35,24 & 34,04 & 7293 & 42,74 & 44,04 & 2784 & 50,32 & 46,4 \\
\hline \multicolumn{2}{|c|}{ Zone $6 \ldots \ldots \ldots$} & 12175 & 34,56 & 37,78 & 2125 & 42,21 & 45,65 & 1831 & 65,27 & 60,1 \\
\hline $\begin{array}{l}\text { Type de } \\
\text { peuplement }\end{array}$ & $\mathrm{MD}$ & $\begin{array}{r}216990 \\
35619 \\
133997\end{array}$ & & & $\begin{array}{ll}29 & 326 \\
11 & 147 \\
10 & 645\end{array}$ & $\begin{array}{l}24,46 \\
30,49 \\
40,32\end{array}$ & $\begin{array}{l}24,30 \\
33,64 \\
38,59\end{array}$ & $\begin{array}{r}131893 \\
4368 \\
22066\end{array}$ & & $\begin{array}{l}21,8 \\
66,9 \\
37,4\end{array}$ \\
\hline \multicolumn{2}{|c|}{ Ensemble du massif } & 386606 & 15,04 & 13,59 & 51119 & 17,65 & 17,69 & 158327 & 20,50 & 18,98 \\
\hline
\end{tabular}




\section{Tableau 5}

Estimations des variations de population ( $D=T_{n}-T_{n-1}$ ) entre cycles consécutifs suivis de leurs coefficients de variation en $p .100$, sans tenir compte du fait que les observations sont faites sur les mêmes pins puis en en tenant compte, par zone, par type de peuplement et pour l'ensemble du massif de 1971 à 1982.

\begin{tabular}{|c|c|c|c|c|c|c|c|}
\hline \multirow{2}{*}{\multicolumn{2}{|c|}{ Zone $1 \ldots \ldots \ldots \ldots$}} & \multicolumn{3}{|c|}{1971 à 1972} & \multicolumn{3}{|c|}{1972 à 1973} \\
\hline & & -86683 & 31,82 & 24,69 & -49249 & 19,81 & 19,42 \\
\hline \multicolumn{2}{|l|}{ Zone 2} & -196499 & 23,81 & 12,99 & -154671 & 13,37 & 12,95 \\
\hline \multicolumn{2}{|l|}{ Zone 3} & -51215 & 59,44 & 45,73 & -43059 & 35,31 & 32,80 \\
\hline \multicolumn{2}{|l|}{ Zone 4} & -19407 & 101,23 & 54,00 & -23056 & 37,84 & 37,78 \\
\hline \multicolumn{2}{|l|}{ Zone 5} & - 8442 & 125,39 & 65,62 & -14456 & 38,39 & 27,92 \\
\hline \multicolumn{2}{|l|}{ Zone 6} & - 4287 & 93,84 & 67,59 & $-\quad 3969$ & 29,35 & 28,63 \\
\hline $\begin{array}{l}\text { Type } \\
\text { de peuplement }\end{array}$ & $\begin{array}{l}\mathrm{C} \ldots \\
\mathrm{MD} \\
\mathrm{D} .\end{array}$ & $\begin{array}{r}-191670 \\
-\quad 64225 \\
-110640 \\
\end{array}$ & $\begin{array}{l}24,43 \\
43,63 \\
33,97 \\
\end{array}$ & $\begin{array}{l}16,25 \\
10,41 \\
25,43\end{array}$ & $\begin{array}{r}-152786 \\
-\quad 60024 \\
-\quad 75649 \\
\end{array}$ & $\begin{array}{l}14,28 \\
18,11 \\
21,63 \\
\end{array}$ & $\begin{array}{l}13,19 \\
18,11 \\
21,36\end{array}$ \\
\hline \multicolumn{2}{|c|}{ Ensemble du massif } & -366535 & 18,08 & 11,60 & -288459 & 10,18 & 9,72 \\
\hline
\end{tabular}

Tableau 5 (suite)

\begin{tabular}{|c|c|c|c|c|c|c|c|}
\hline \multirow{2}{*}{\multicolumn{2}{|c|}{ Zone 1}} & \multicolumn{3}{|c|}{1973 à 1974} & \multicolumn{3}{|c|}{1974 à 1975} \\
\hline & & 27319 & 28,02 & 27,67 & -18914 & 42,47 & 37,67 \\
\hline Zone $2 \ldots \ldots$ & & 56669 & 22,68 & 22,10 & -61843 & 20,50 & 18,26 \\
\hline Zone 3 & & 16762 & 52,39 & 51,86 & -11038 & 92,98 & 78,85 \\
\hline Zone 4 & & 8636 & 94,33 & 94,34 & - 10997 & 72,00 & 72,00 \\
\hline Zone $5 \ldots$ & & 2522 & 122,13 & 122,12 & - 4022 & 62,93 & 59,11 \\
\hline Zone $6 \quad \ldots \ldots$ & & 3769 & 60.20 & 59,98 & -2138 & 127,50 & 50,11 \\
\hline $\begin{array}{l}\text { Type } \\
\text { de peuplement }\end{array}$ & $\begin{array}{l}\mathrm{C} \ldots \\
\mathrm{MD} . \\
\mathrm{D} \ldots\end{array}$ & $\begin{array}{l}62492 \\
16518 \\
36667 \\
\end{array}$ & $\begin{array}{l}22,18 \\
30,40 \\
34,99\end{array}$ & $\begin{array}{l}21,54 \\
30,40 \\
34,94 \\
\end{array}$ & $\begin{array}{l}66897 \\
-\quad 14402 \\
-\quad 27653\end{array}$ & $\begin{array}{l}20,18 \\
36,36 \\
50,80\end{array}$ & $\begin{array}{l}18,91 \\
36,57 \\
42,18\end{array}$ \\
\hline \multicolumn{2}{|c|}{ Ensemble du massif } & 115676 & $16, \% 0$ & 16,64 & -108951 & 18,52 & 16,52 \\
\hline
\end{tabular}


TABLEAU 5 (suite)

Estimates of population variations $\left(D=T_{n}-T_{n \ldots 1}\right.$ ) between consecutive cycles followed by their coefficients of variation in $\mathrm{p}$. 100, without considering the fact that observations were made on the same pine trees (first table)

and then considering this factor (second table), in each zone, each stand type, and for the whole area from 1971 to 1982.

\begin{tabular}{|c|c|c|c|c|c|c|c|}
\hline \multirow{2}{*}{\multicolumn{2}{|c|}{ Zone $1 \ldots \ldots \ldots \ldots$}} & \multicolumn{3}{|c|}{1975 à 1976} & \multicolumn{3}{|c|}{1976 à 1977} \\
\hline & & 5559 & 85,12 & 66,32 & 53086 & 25,35 & 22,18 \\
\hline \multicolumn{2}{|l|}{ Zone 2} & 9745 & 53,25 & 52,66 & 41662 & 26,93 & 24,88 \\
\hline \multicolumn{2}{|c|}{ Zone $3 \ldots \ldots \ldots \ldots$} & -1492 & 451,74 & 382,29 & 15770 & 57,65 & 45,45 \\
\hline \multicolumn{2}{|l|}{ Zone 4} & 3927 & 99,85 & 99.86 & 1933 & 264,92 & 219,23 \\
\hline \multicolumn{2}{|l|}{ Zone 5} & 1547 & 74,85 & 75,05 & 5522 & 48,84 & 43,14 \\
\hline \multicolumn{2}{|l|}{ Zone 6} & 400 & 474,25 & 340,36 & 2963 & 104,08 & 67,21 \\
\hline $\begin{array}{l}\text { Type } \\
\text { de peuplement }\end{array}$ & $\begin{array}{l}\text { C . } \\
M D \\
D \ldots\end{array}$ & $\begin{array}{r}25360 \\
2252 \\
-7926\end{array}$ & $\begin{array}{r}29,24 \\
138,54 \\
89,53\end{array}$ & $\begin{array}{r}26,02 \\
144,25 \\
76,67\end{array}$ & $\begin{array}{r}88408 \\
7459 \\
25068\end{array}$ & $\begin{array}{l}20,97 \\
63,41 \\
32,58\end{array}$ & $\begin{array}{l}17,80 \\
70,67 \\
28,15\end{array}$ \\
\hline \multicolumn{2}{|c|}{ Ensemble du massif .... } & 19686 & 54,49 & 48.47 & 120934 & 17,20 & 14,91 \\
\hline
\end{tabular}

Tabliau 5 (suite)

\begin{tabular}{|c|c|c|c|c|c|c|c|}
\hline \multirow{2}{*}{\multicolumn{2}{|c|}{ Zone 1}} & \multicolumn{3}{|c|}{1977 à 1978} & \multicolumn{3}{|c|}{1978 à 1979} \\
\hline & & 130987 & 23,29 & 16,06 & -31070 & 123,28 & 108,54 \\
\hline \multicolumn{2}{|l|}{ Zone 2} & 143941 & 23,81 & 17,45 & -202050 & 16,21 & 16,21 \\
\hline \multicolumn{2}{|l|}{ Zone 3} & 68058 & 34,66 & 21,87 & 33769 & 136,95 & 110,48 \\
\hline \multicolumn{2}{|l|}{ Zone 4} & 13902 & 67,81 & 58,47 & 22553 & 77,15 & 42,46 \\
\hline \multicolumn{2}{|l|}{ Zone 5} & 7650 & 87,07 & 61,61 & 22137 & 63,62 & 34,58 \\
\hline \multicolumn{2}{|l|}{ Zone 6} & 6319 & 75,34 & 25,59 & 756 & 789,42 & 410,93 \\
\hline $\begin{array}{l}\text { Type } \\
\text { de peuplement }\end{array}$ & $\begin{array}{l}\mathrm{C} \\
\mathrm{MD} \\
\mathrm{D}\end{array}$ & $\begin{array}{r}233732 \\
57854 \\
79271\end{array}$ & $\begin{array}{l}19,47 \\
28,74 \\
27,47\end{array}$ & $\begin{array}{l}13,12 \\
24,49 \\
19,77\end{array}$ & $\begin{array}{r}-143800 \\
-\quad 34062 \\
23957\end{array}$ & $\begin{array}{r}37,80 \\
57,16 \\
181,00\end{array}$ & $\begin{array}{l}29,29 \\
59,19 \\
64,67\end{array}$ \\
\hline \multicolumn{2}{|c|}{ Ensemble du massif .... } & 370857 & 14,32 & 10,04 & -15904 & 46,92 & 31,97 \\
\hline
\end{tabular}


Tableau 5 (suite)

\begin{tabular}{|c|c|c|c|c|c|c|c|}
\hline \multirow{2}{*}{\multicolumn{2}{|c|}{ Zone $1 \ldots \ldots \ldots \ldots \ldots$}} & \multicolumn{3}{|c|}{1979 à 1980} & \multicolumn{3}{|c|}{1980 à 1982} \\
\hline & & -150245 & 18,03 & 16,82 & 40116 & 32,25 & 29,34 \\
\hline \multicolumn{2}{|l|}{ Zone 2} & 9868 & 43,99 & 43,99 & 35760 & 51,00 & 39,83 \\
\hline \multicolumn{2}{|c|}{ Zone $3 \ldots \ldots \ldots \ldots$} & -118261 & 34,46 & 33,55 & 25816 & 56,53 & 54,60 \\
\hline \multicolumn{2}{|l|}{ Zone 4} & -36928 & 41,69 & 33,71 & 10319 & 152,72 & 116,50 \\
\hline \multicolumn{2}{|l|}{ Zone 5} & -29872 & 43,69 & 36,05 & -4508 & 76,82 & 63,53 \\
\hline \multicolumn{2}{|l|}{ Zone 6} & -10050 & 46,78 & 39,80 & - 294 & 499,32 & 429,13 \\
\hline $\begin{array}{l}\text { Type } \\
\text { de peuplement }\end{array}$ & $\begin{array}{l}\mathrm{C} \ldots \\
\mathrm{MD} . \\
\mathrm{D} .\end{array}$ & $\begin{array}{r}-187664 \\
-\quad 24472 \\
-123352\end{array}$ & $\begin{array}{l}18,70 \\
46,85 \\
31,18\end{array}$ & $\begin{array}{l}16,64 \\
42,80 \\
30,71\end{array}$ & $\begin{array}{r}102567 \\
-6779 \\
11421\end{array}$ & $\begin{array}{l}28,88 \\
70,13 \\
80,84\end{array}$ & $\begin{array}{l}23,90 \\
60,66 \\
76,55\end{array}$ \\
\hline \multicolumn{2}{|c|}{ Ensemble du massif } & -335487 & 15,89 & 14,96 & 107208 & 29,28 & 24,58 \\
\hline
\end{tabular}

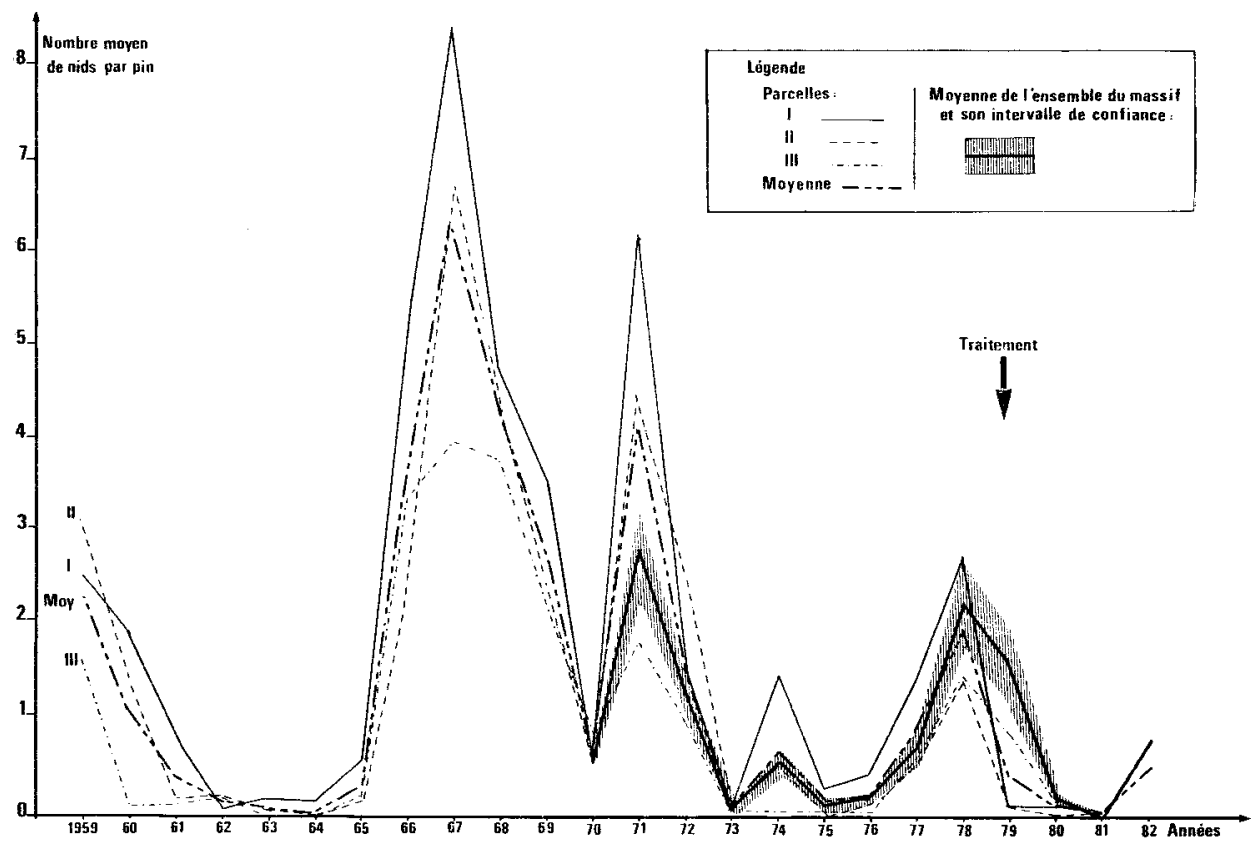

FIG. 2

Nombres moyens de nids par pin dans les parcelles de dénombrement de l'O.N.F. Comparaison aux estimations obtenues depuis 1970 pour l'ensemble du massif. Mean number of nests per pine in O.N.F. counting plots, compared with estimates of the whole area since 1970. 


\subsection{Examen comparatif des dénombrements en parcelle effectués par l'O.N.F.}

La comparaison entre le nombre moyen de nids par pin dans les 3 parcelles de dénombrement des forestiers et dans l'ensemble du massif d'après notre dispositif d'échantillonnage (fig. 2) montre une bonne correspondance jusqu'en 1978 ainsi qu'une grande similitude des fluctuations. Après 1979, l'écart est plus important, deux parcelles étant situées dans la zone traitée. Des valeurs à nouveau comparables en 1982 montrent la remontée des populations dans les sites traités ou non.

Les moyennes parcellaires fournissent donc une image assez fidèle des variations des populations du massif et peuvent être utilisées pour une étude rétrospective de leur évolution antérieure à l'étude. Elles ne sont toutefois pas absolument représentatives de ces populations. Avant 1973, période de fortes populations dans la zone 2, elles sont souvent hors de l'intervalle de confiance des moyennes du massif qu'elles surestiment. De tels biais ont pu avoir lieu antérieurement.

\subsection{Discussion}

La figure 2 montre une régression des populations suivie d'une longue période de latence après le traitement de 1959 , puis une rapide réinfestation culminant en 1967, une nouvelle régression avec reprise temporaire de l'infestation en 1970 et 1974 , une courte phase de latence et un nouvel accroissement interrompu par le traitement de 1979. Ce traitement est lui-même suivi d'une régression générale et d'une troisième phase de croissance.

Cette évolution a un caractère relativement cyclique avec une périodicité assez mal définie ( 8 à 10 ans ?), beaucoup moins régulière que pour la Tordeuse du Mélèze (Auer, 1961, 1968). Les phases de croissance et de décroissance sont perturbées par des variations apériodiques, qui peuvent dépendre de facteurs tels que le climat, la diapause et l'impact des traitements.

La phase de latence observée après le traitement à base de virus réalisé en 1958-1959 ne s'cst pas reproduite par la suite. Peut-être était-elle une conséquence de cette intervention. Le traitement de 1979, outre son effet local immédiat, a pu avoir à plus long terme une incidence indirecte sur la décroissance et la remontée des populations.

Quelles que soient les fluctuations enregistrées la répartition des populations n'a subi que de faibles modifications; les observations effectuées dans d'autres massifs. forestiers (GERI, 1971, 1980 a et b, 1983) sont confirmées; les plus fortes infestations demeurent localisées au sud et dans les peuplements clairs, les plus faibles au nord et dans les peuplements denses.

Il semble toutefois que chaque progression affecte en premier lieu les peuplements clairs, tandis que des régressions plus tardives sont observées dans les peuplements denses. Des variations des effectifs relatifs des zones sont en outre enregistrées.

Malgré celles-ci, on peut raisonnablement affirmer que les zones non traitées ont valablement servi de témoin à la zone 2 pour le traitement le 1979. 


\section{Etude des relations entre les populations et leur milieu}

\subsection{Corrélations et tableaux de contingence}

Les corrélations simples confirment ces relations entre l'insecte et le milieu. Des corrélations significatives sont obtenues entre les mesures de population et :

- l'altitude (positives), la pente (négatives),

- l'exposition (positives pour le sud, négatives pour le nord),

- le nombre de pins par placette, la densité des peuplements (négatives),

- la taille des pins échantillonnés, la hauteur de l'étage principal (négatives).

Des corrélations de signe opposé lient ces facteurs au nombre d'années sans attaques.

Aucune relation n'est pratiquement enregistrée avec le nombre d'étages et le diamètre des pins et peu sont notées entre les populations et la dominance. Le pin sylvestre, ćchantillonné ou présent dans le peuplement correspond souvent à des populations plus faibles, le mélange avec des feuillus ou des cèdres à des populations plus fortes.

Les liaisons des variations de population entre années consécutives avec ces facteurs sont très semblables à celles des populations elles-mêmes lorsque les populations s'accroissent (1973 à 1974, 1975 à 1978) mais s'inversent en période de décroissance (1971 à 1972, 1974 à 1975).

En outre, les différentes mesures de population sont très liées entre elles, pour chaque année, ce qui confirme la validité des indices de population utilisés, et d'une annéc à l'autre, ce qui met en évidence le maintien de la répartition de linfestation au cours de la gradation, tant au niveau du pin que des zones et sous-zones. On note en particulier une grande similitude entre la répartition des populations de 1971, de 1972 et de 1974 (alors que la population de 1974 est peu liée à celle de 1973), peut-être due à un apport en 1974 d'individus diapausants s'étant nymphosé en 1971 et en 1972.

Les variations de population sont lices positivement aux nombres de nids de chaque année et à celui de l'ensemble des années (variable TOTAL) lorsqu'elles traduisent un accroissement, négativement dans le cas contraire.

Ces corrélations suggèrent que les populations sont réparties dans le massif en fonction d'un schéma d'autant plus strict que les populations sont plus importantes et qui devient moins apparent durant les phases de régression.

Les coefficients de corrélation les plus élevés sont observés pour les années de forte infestation. Un nombre plus limitê de significations est obtenu en considérant les variations de population sous forme de rapport que sous forme de différence, mais elles caractérisent vraisemblablement mieux l'évolution des populations (la différence devient très semblable à la population elle-même quand l'effectif d'une année est grand). On note des corrélations négatives pouvant traduire des phénomènes de dépendance de la densité après de fortes attaques : variation de population de 1971 à 1972 par rapport à la population de 1971 , de 1977 à 1978 par rapport à celle de 1977. 
Ces résultats confirment les relations observées dans d'autres massifs, en particulier pour l'exposition et la densité des peuplements. Certaines liaisons sont plus spécifiques des pineraies du Ventoux et dépendent des relaltions entre les facteurs du milieu propre à ce massif. Celles-ci sont mises en évidence par les corrélations entre variables du milieu et par le tableau de contingence de ces variables sous forme qualitative (tabl. 2) qui montre en outre la diversité de la répartition de ces facteurs dans les différentes zones. Ainsi la corrélation entre le nombre de nids et la taille des pins est négative et non positive; mais les boisements de petite taille occupent des sites clairiérés, où les arbres croissent lentement, et la hauteur des arbres est fortement liéc à la densité. La liaison négative avec la pente résulte vraisemblablement de la corrélation positive de celle-ci avec les expositions nord. L'incidence éventuelle des différentes essences sur l'infestation, est masquée par leur localisation : les pins sylvestres (corrélations négatives) sont plus nombreux dans les boisements denses, les feuillus et les cèdres (corrélations positives) dans les espaces clairs exposés au sud. Enfin la faible diversification du nombre d'étages dans cette forêt récente explique l'absence de corrélations avec ce facteur.

Ces corrélations, qui illustrent l'étroite dépendance entre les variables du milieu, montrent la nécessité d'apprécier leur incidence propre.

\subsection{Analyses de variance et de covariance non orthogonales à plusieurs facteurs}

\subsection{Incidence des facteurs du milieu forestier}

L'analyse de variance non orthogonale a été effectuće sur les populations des années 1971 à 1982 ; en considérant les corrélations et les résultats d'une première étude (GERI, 1980 a), les variables $Z$ (logarithme du nombre de nids +1 ) et I pin de chaque année et les variables «TOTAL et «ANNEES》 (\$4.222.) ont été choisies comme variables expliquées, les variables altitude, exposition, hauteur de l'étage principal, densité du peuplement, essence (pin noir ou pin sylvestre), comme variables explicatives, avec les classes du tableau 1 (pour avoir des effectifs suffisants, les pins isolés ont été regroupés avec les peuplements clairs).

Toutes les interactions ont d'abord ćté testées sur la variable «TOTAL». Seules les interactions significatives, densité $\times$ essence, et altitude $\times$ exposition, ont été conservécs.

Les résultats obtenus pour $Z$, «TOTAL » et «ANNEES » sont présentés (tabl. 6). Toutes les carastéristiques du milieu forestier, exceptée la hauteur de l'étage principal, significative au seuil 5 p. 100 , sont hautement significatives vis-à-vis de «TOTAL 》 et « ANNEES»; elles sont souvent significatives vis-à-vis des variables $Z$ des différentes années. Les significations les plus nettes sont notées pour l'essence, l'exposition et la densité. Les interactions ne sont que rarement significatives.

Lincidence de l'altitude est nette en 1971, 1972 et 1974, elle disparaît ensuite; l'effet de l'exposition marqué en 1971 n’apparaît pas en 1972. La densité des peuplements et l'essence sont les facteurs les plus régulièrement liés à l'importance des populations. Peu de significations sont observées pour les faibles populations (1973, 1975, 1976). 


\section{Tableau 6}

Analyse de variance non orthogonale.

$Z_{n}, Z_{n}-Z_{n-1}$, «TOTAL» et «ANNEES» en fonction des facteurs du milieu forestier. Tableat des $F$ et de leurs significations.

Non-orthogonal variance analysis.

$Z_{n}, Z_{n}-Z_{n-1}$, «TOTAL》 and «ANNEES》 as a function of the forest environmental factors. Table of $F$ and their significance.

\begin{tabular}{|c|c|c|c|c|c|c|c|c|}
\hline & ALT. & EXP. & PL. HT. & DENS. & DOM. & ESS. & $\begin{array}{c}\text { DENS. } \\
\times \\
\text { ESS. }\end{array}$ & $\begin{array}{c}\text { ALT. } \\
\times \\
\text { EXP. }\end{array}$ \\
\hline & $1,464 * *$ &, $438 * \cdots$ & $6,114 * *$ & $9,213 * *$ & 3,531 & $15,159 * *$ & 0.933 & 1,663 \\
\hline & $10,742 * *$ & 1,267 & $3,929 *$ & $12,895^{* *}$ & $11,163^{* *}$ & $14,6,48^{*} *$ & 1.221 & $1,889 *$ \\
\hline- & 1,079 & 1,278 & 1,023 & 2,572 & 2,578 & 1,236 & 1,648 & 0,612 \\
\hline & $6,328 * *$ & $4,350^{* *}$ & 2,030 & $3,742^{*}$ & 0,549 & $6,007 *$ & 2,514 & 1,618 \\
\hline & 1,547 & $4,673 *$ & $4,191^{* *}$ & 0,459 & 0,243 & 0,034 & 1,398 & 0,777 \\
\hline & 1,58 & 2,006 & $3,159 * *$ & $3,069^{*}$ & 1,3 & 2,344 & 1,310 & 0,802 \\
\hline & 1,906 & $3,711 * *$ & 1,096 & $14,392: *$ & $4,168^{*}$ & $15,641 * *$ & $5,222 * *$ & 0,572 \\
\hline & 1,192 & $11,111 * *$ & 2,562 & $17,225 * *$ & $9,093 * *$ & $32,272 *$ & $4,588 *$ & 0,723 \\
\hline$Z_{1979}$ & 6,67 & $2,692 *$ & 1,510 & $7,660^{*}$ & 2,566 & $7,069^{* * *}$ & 2,550 & $1,837^{*}$ \\
\hline & 2,025 & 1,269 & 2,454 & $11,570^{*} *$ & $5,086^{*}$ & $5,021^{*}$ & 1,913 & $2,049^{*}$ \\
\hline$Z_{19 k 2}$ & 0,486 & $4,973 * *$ & $3,135^{*}$ & $12,086 \% *$ & 0.292 & $4,128^{*}$ & $4,060^{*}$ & 0,630 \\
\hline$Z_{1972}-Z_{1}$ & & $6 * *$ & & & & & 0,059 & 1,060 \\
\hline & & $\begin{array}{l}0,4 \\
\end{array}$ & & $8,589 * *$ & $7,054 * *$ & $11,008 * *$ & 0,476 & 1,522 \\
\hline $\mathrm{Z}_{1974}-\mathrm{Z}_{1973}$ & 3 & 2,333 & 1,912 & 1,275 & 0,000 & 3,250 & 0,928 & 1,237 \\
\hline $7=-7$ & $611 *$ & 1,746 & 1,310 & $5,189 * *$ & 0,313 & $6,736 * *$ & 1,346 & 1,837 * \\
\hline$Z_{1976}-Z_{1 !}$ & $2,952 *$ & 0,426 & 0,599 & $2,997 *$ & 0,606 & 1,775 & 0,438 & 0,608 \\
\hline$Z_{1977}-Z_{1 !}$ & 1,133 & 1,423 & 0,413 & $7,897^{*} *$ & 1,880 & $9,747 * *$ & 2,710 & 0,623 \\
\hline & & $7,393 * *$ & 1,840 & $3,226^{*}$ & 3,708 & $12,406^{* *}$ & 0,669 & 1,047 \\
\hline$Z_{1979^{-}} Z_{19}$ & 7,019 & $4,702 * *$ & 1,594 & 1,350 & 1,372 & $6,425^{*}$ & $3,150^{*}$ & $2,353^{*}$ \\
\hline$Z_{1981}-Z_{197 !}$ & $7,730)^{*: / k}$ & & & 2,521 & & 3,087 & 2,194 & 1,383 \\
\hline$Z_{1982}-Z_{1980}$ & 2,371 & $4,111 * *$ & $4,677 * *$ & 1,962 & 0,827 & 0,376 & 1,367 & 1,209 \\
\hline & & & & & & & $4,459^{*}$ & 1,033 \\
\hline Années & $5,912 * *$ & $|13,761 * *|$ & $3,450 *$ & $14,945^{* *}$ & $7,802 * *$ & $|24,825 * *|$ & 2,126 & 1.110 \\
\hline
\end{tabular}

Abbréviations

ALT. : altitude.

EXP. : exposition.

PL.H.T. : hauteur de l'étage principal.

DENS. : densité.

DOM. : dominance.

ESS. : essence.

* : Significatif au seuil 5 p. 100.

* : Significatif au seuil 1 p. 100 . 

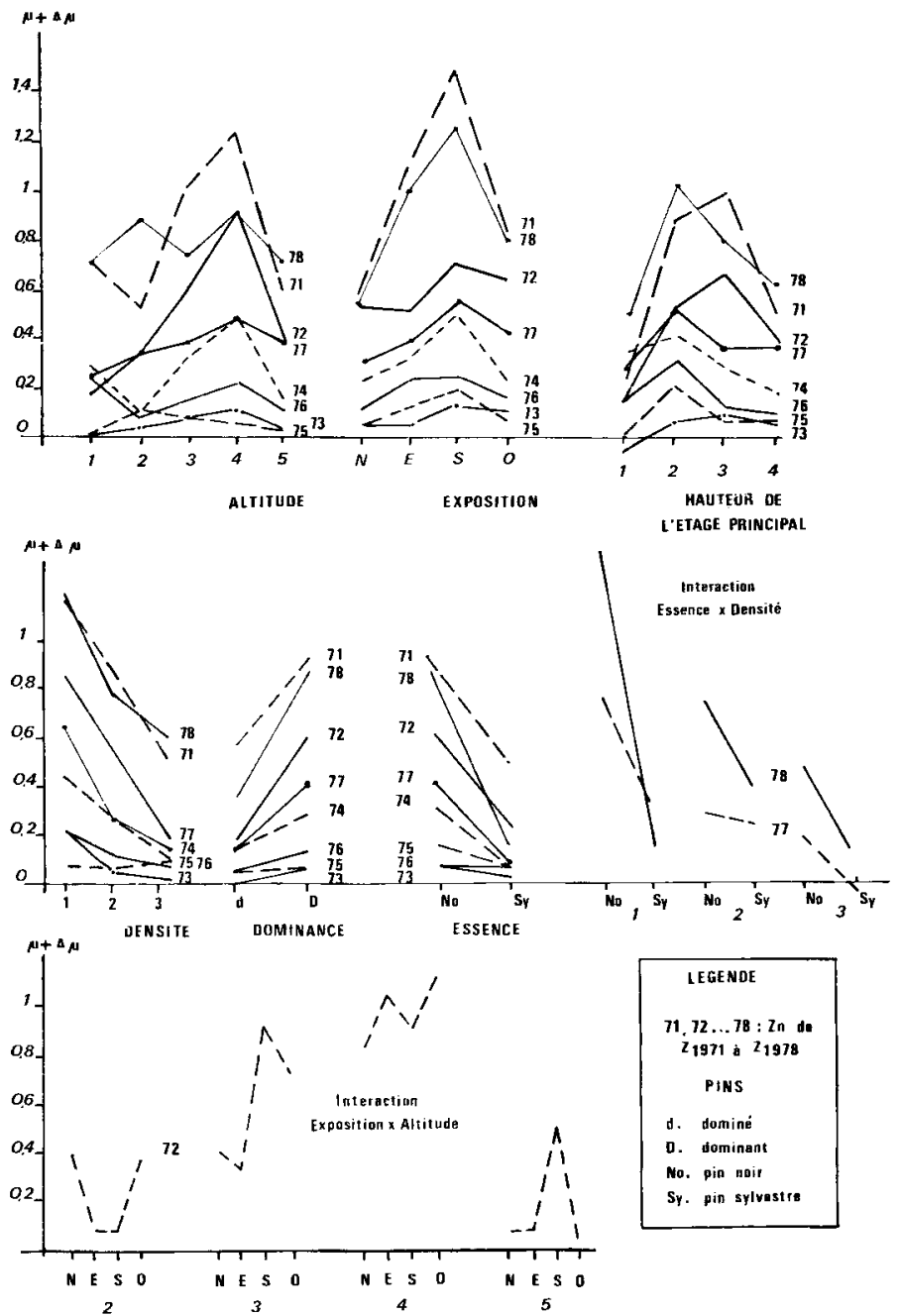

FIG. 3

Analyse de variance non orthogonale. Etude de la Variable $Z_{n}$. $\mu+\Delta \mu$ en fonction des différentes classes de facteurs du milieu.

Non orthogonal variance analysis.

On variable $Z_{n}, \mu+\Delta u$ according to various classes of environmental factors. 
Des résultats très voisins sont obtenus avec «I pin».

La forme de ces relations est présentée pour la variable $\mathrm{Z}$ jusqu'en 1978 sur la figure 3 :

- L'infestation croît jusqu’à $700-800 \mathrm{~m}$, décroît au-dessus. Cette relation n'est plus très apparente après 1975. A cet égard 1978, annéc de forte population se distingue nettement de 1971.

- Les populations sont plus importantes sur les pins exposés au sud que sur ceux exposés au nord. Les expositions est et ouest ont des valeurs intermédiaires. Cet effet, très net en 1971, disparaît en 1982, ce qui montre une régression importante dans les sites bien exposés; il se rétablit ensuite.

- Les pcuplements de taille moyenne $(6$ à $12 \mathrm{~m}$ jusqu'en 1973, 4 à $6 \mathrm{~m}$ ensuite) sont les plus infestés.

- Le nombre de nids décroît avec la densité des boisements.

- Les arbres dominants sont plus pcuplés que les dominés.

- Les pins noirs sont plus attaqués que les pins sylvestres.

Pour les interactions, seules les liaisons significatives sont représentées; la différence entre pin sylvestre et pin noir est accrue dans les boisements clairs. En 1972, les populations sont particulièrement réduites en exposition sud en basse altitude.

Des rclations très semblables sont observées avec «I pin».

Il n’a pas été tenu compte sur la figure 3 des données postérieures au traitement de 1979. Son effet se traduit surtout par une diminution des populations dans des expositions sud et est et des régions d’altitude moyenne.

Cette analyse confirme bien lexistence de relations entre les populations et la structure de l'environnement forestier ainsi que la généralité de l'influence de la densité, de l'exposition, de la dominance et des essences, observée dans d'autres massifs (C. Geri, 1980 a, b ; 1983). Elle précise la forme souvent non linéaire de ces relations. Certaines paraissent plus spécifiques du Ventoux. Ainsi la décroissance des populations au-dessus de $800 \mathrm{~m}$ peut s'expliquer par le climat (\$2.2.). La diminution de l'infestation sur les grands pins, notée dans d’autres massifs mais très marquée au Ventoux, est réduite quand on prend en compte les autres facteurs du peuplement dans le modèle. Elle résulte en partie des conditions particulières de ce massif ( $\$ 2.1$.).

L'analyse fait, en outre, apparaître des modifications de ces relations dannée en année qui méritent d'être approfondies.

\subsection{Prise en compte des niveaux de populations antérieurs; analyse de covariance non orthogonale}

Cette analyse, dérive de la précédente en introduisant la covariance entre la population étudiée et la population de l'année précédente; elle a été réalisce antérieurement sur la variable $Z$ et sur les mêmes variables du milieu à l'exclusion de l'essence et en prenant en compte le nombre d'étages. vantes :

En ne considérant que les facteurs significatifs, on a obtenu les équations sui- 
ลลกิ์

总总总总总总

농ㅇㅇㅇㅇㅇ

00000

营灾

$\stackrel{\infty}{0} 8$

$+1$

สิิศิซ

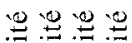

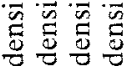

整

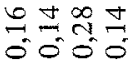

$1+1 \mid$

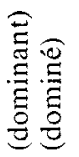

mis

00

$+1$

$+$

สลิสซิ

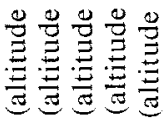

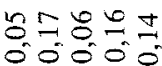

$11++1$

콩쿄

형

$1+$

$+$

สลิกษ

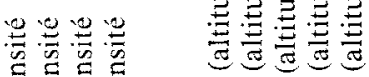

氖导导

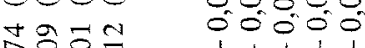

당요 $1+111$

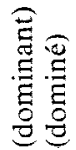

용

$+1$

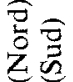

5.

$1+$

ลลกิซ $\quad+$

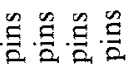

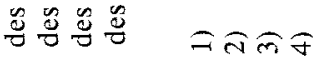

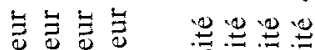

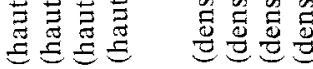

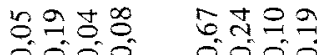

0000 000

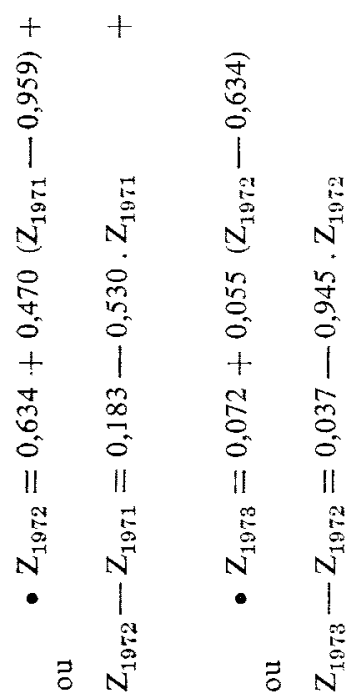<smiles>COC#N</smiles><smiles>C=C=[Te]</smiles>

$\stackrel{+}{+\infty}$

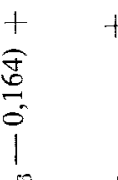

畄

N

$\stackrel{18}{9}$

N

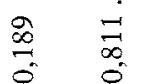

in

$\begin{array}{ll}+ & 1 \\ 0 & 8 \\ 0 & 0\end{array}$

$+$

苞

II 1

뇽

ว

II

골

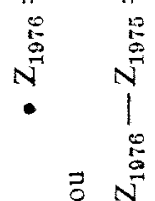

$\infty$
0
0

$+$

ह $\frac{1}{2}$

o

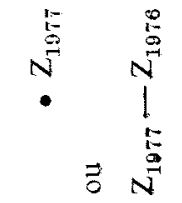


Elles confirment, au niveau des pins, la liaison entre les populations d'annéc en année. Le fait que le coefficient de régression soit souvent très inférieur à 1 , surtout observé lorsque les populations s'abaissent à de très faibles niveaux (1973) mais moins marqué pendant les périodes de croissance (1975 à 1977), peut traduire des effets négatifs des niveaux de population antérieurs. Toutefois, l'interprétation de ces coefficients de régression doit être prudente. Dans la mesure où la population de chaque arbre est renouvelée à chaque cycle on ne peut en effet raisonner dans un cadre strict de dépendance de la densité comme dans le modèle de Morris (1963).

Ces équations montrent en outre que les facteurs du milieu (densité, exposition, altitude, hauteur des pins, dominance) ont encore une action lorsque la population du cycle précédent a été prise en considération. Durant les périodes de croissance des populations (1973 à 1974, 1975 à 1977), ils interviennent sur les variations de populations comme sur les populations elles-mêmes, renforçant d'année en année la liaison entre populations et facteurs du milieu. Des effets opposés sont notés en phase de régression, après 1971 pour la densité, de 1971 à 1972 pour l'exposition. Jusqu'en 1975, les populations se maintiennent mieux dans les régions d'altitude moyenne.

Ces résultats confortent le schéma d'évolution des liaisons entre les populations et les facteurs du milieu suggéré par les corrélations, mais ne permettent pas une présentation simple de l'incidence de ces facteurs.

\subsection{Prise en compte des niveaux de populations antérieurs et d'autres facteurs de régulation; développement du modèle d'analyse de variance}

Une seconde démarche (\$ 4.222. et 4.223.) a été préféréc dans l'étude définitive, à partir du modèle d'analyse de variance non orthogonalc, pour rendre compte de laction des facteurs du milieu sur les fluctuations de population (équation 3).

Simultanément, on a testé sur les populations et leurs variations, les facteurs «zones» (équation 2), «traitement» de 1979 et «surpopulation » (équations 7 à 11).

- Le tableau 6 montre l'impact des facteurs du milieu sur les variations de $\mathrm{Z}$ d'un cycle à l'autre. De nombreuses relations significatives sont observées pour l'exposition, laltitude, la densité des peuplements et l'essence; leur forme est représentée figure 4. On note, en particulier, la régression importante des populations de 1971 à $1972\left(\mathrm{~F}=16,68^{++}\right)$dans les expositions sud compensée lors de la remontée des populations $(1977,1978)$, ainsi qu'une diminution importante de 1972 à 1973 et de 1974 à 1975 aux altitudes $700-800 \mathrm{~m}$; la repopulation se faisant ensuite préférentiellement dans ces régions et en basse altitude. Le schéma habituel de répartition des populations pour la densité, l'essence et la dominance paraît renforcé lorsque les populations s'accroissent ; l'inverse est constaté lorsqu'elles régressent.

— Le facteur «Zone » est nettement significatif pour les variables "TOTAL», et «ANNEE», ainsi que pour la variable $Z$ et ses variations pour un certain nombre d'années (tabl. 7). Ce facteur a sa signification propre mais n'améliore pas le pouvoir descriptif du modèle, les variances résiduelles étant comparables. Il ne modifie pas sensiblement les relations avec les facteurs du milieu présentées sur la figure 3 . On retrouve, même après avoir tenu compte de ces facteurs, les constatations relatives aux recensements de populations. Les zones de la face sud $(1,2,6)$ demeurent dans lensemble plus infestées que celles de la face nord ( 3 et surtout 4 et 5 ) et la zone 2 très infestée en 1971 et 1972, est moins peuplée par la suite que les zones 1, 3 et, sauf en 1978, 6 (fig. 5). 

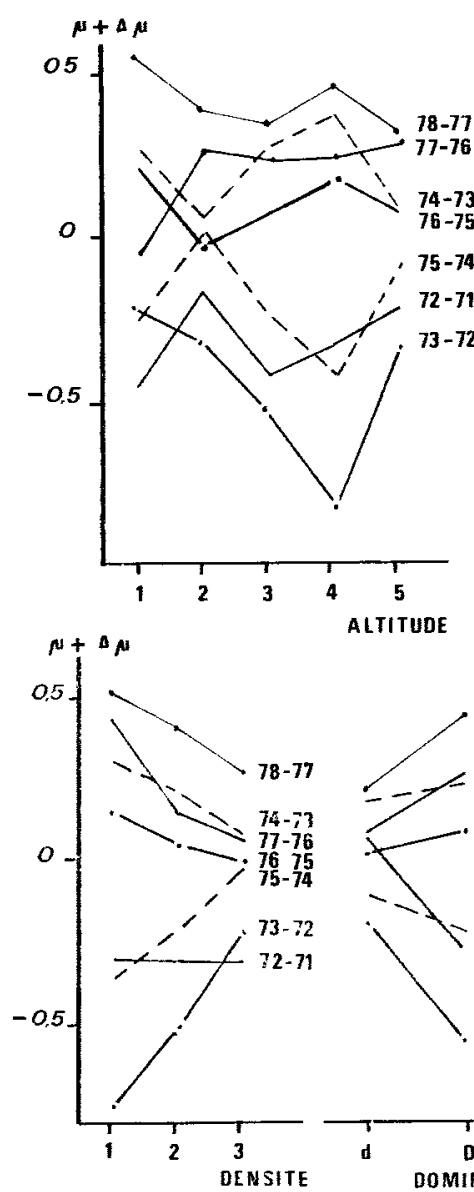
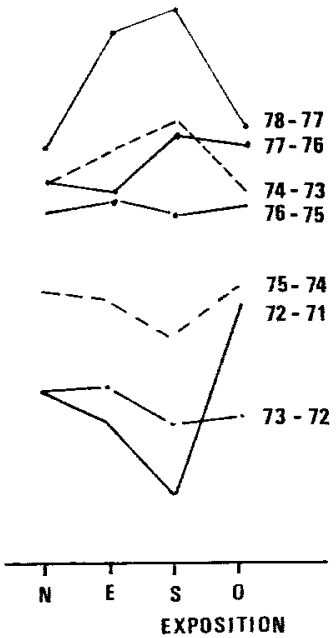

EXPOSITION
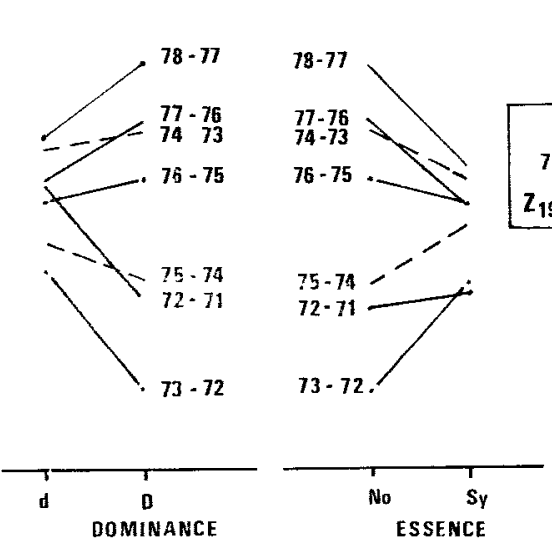

FiG. 4
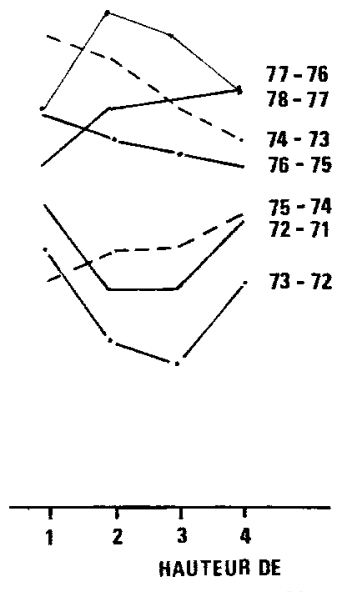

L'ETAGE PMINCIPAL

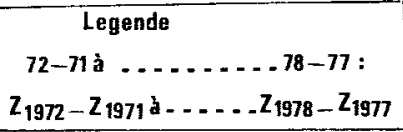

Analyse de variance non orthogonale. Etude de la variable $Z_{n}-Z_{n-1}$ $\mu+\Delta u$ en fonction des différentes classes de facteurs du milieu.

Non orthogonal variance analysis.

On variable $Z_{n}-Z_{n-1}, \mu+\Delta \mu$ according to various classes of environmental factors. 
$\sum_{0}^{\circ}$

$\stackrel{\dot{z}}{2} \times \dot{\vec{x}}$

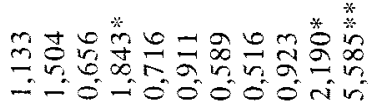

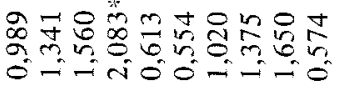
$\begin{array}{ll}2 & 1 \\ \infty & \infty \\ 0 & 0 \\ 0 & 0\end{array}$

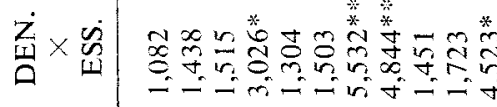

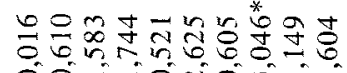
若总 fi

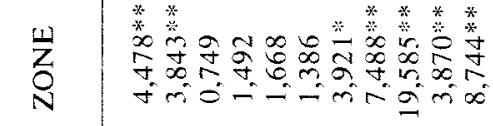

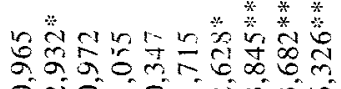
ong-gingo

* in

1

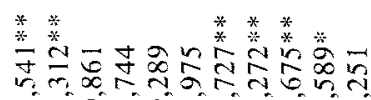

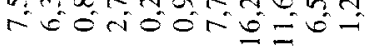

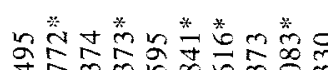

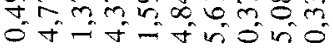

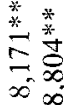

$\dot{n}$

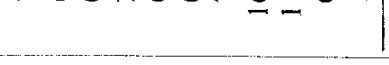

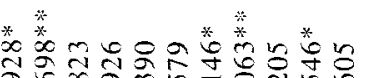

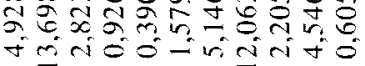

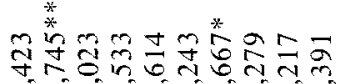
$000004+m 00$

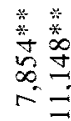

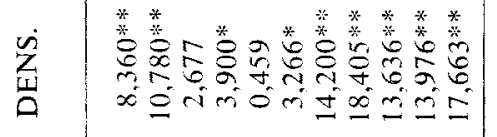

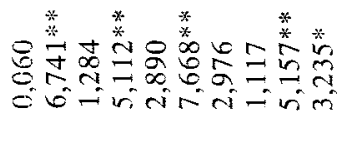
$\stackrel{*}{*}$

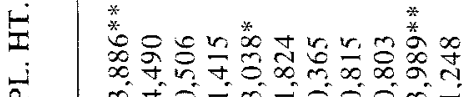

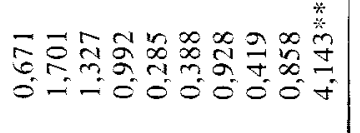
ิㅗㅇ

2

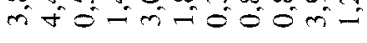

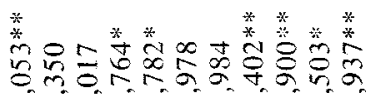

$\underset{2}{2}$ *

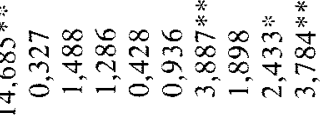

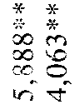
v $\stackrel{5}{*}$

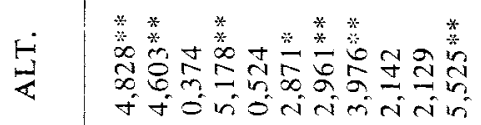

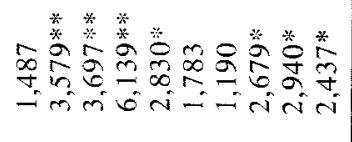



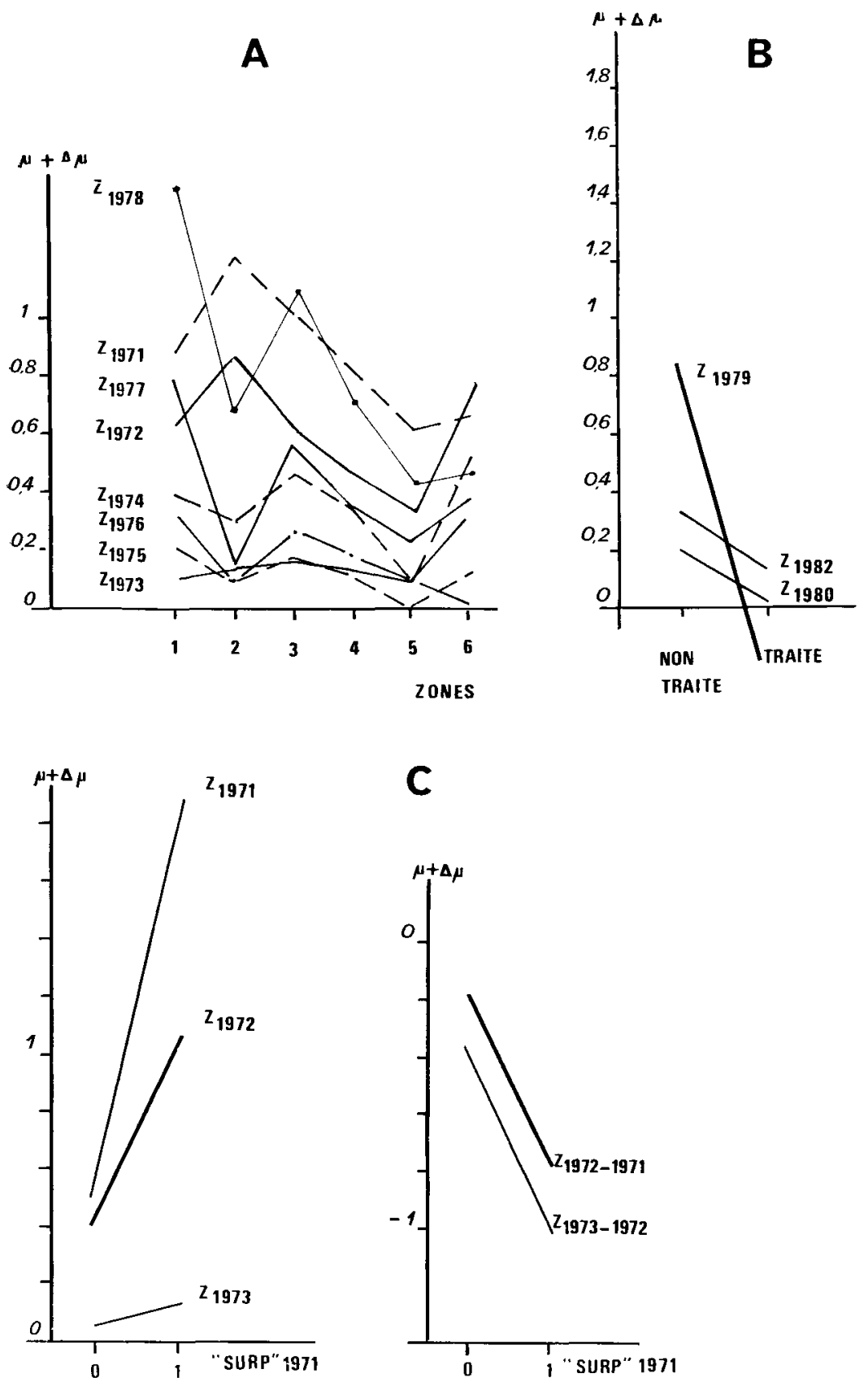

FIG. 5

Analyse de variance non orthogonale.

Incidences du facteur «zone» sur les populations des différentes années $(A)$, du traitement de 1979 sur les populations de 1979 à 1981 (B)

et de la surpopulation de 1971 sur les populations de 1971 à 1973 (C).

Non orthogonal variance analysis.

Effects of «zone»factor on populations of different years (A), of treatment in 1979 on populations from 1979 to 1981

and of overpopulation in 1971 on populations from 1971 to 1973 (C). 


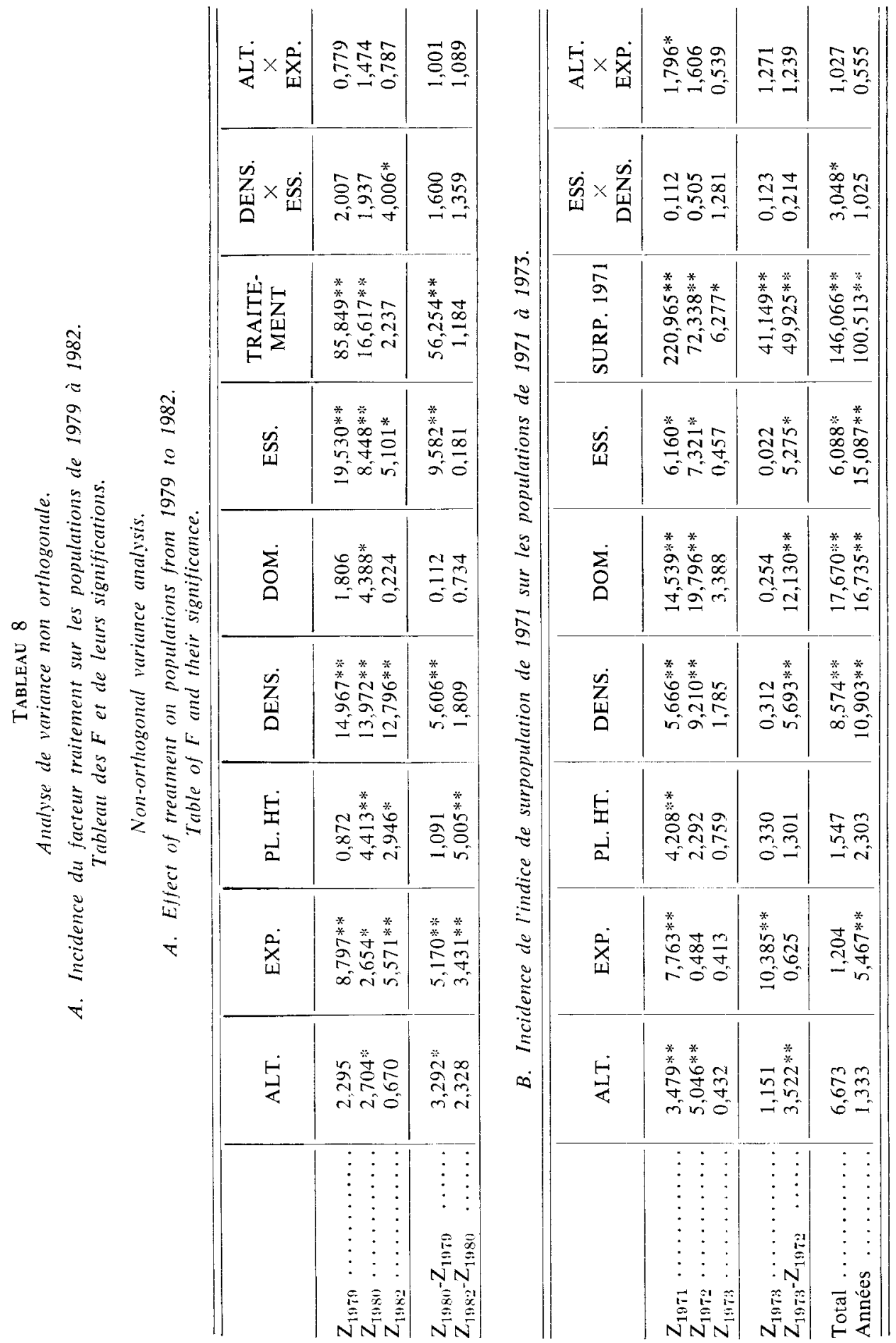


- L'incidence du facteur «Traitement» est très significative sur la variable $\mathrm{Z}$ en $1979\left(\mathrm{~F}=85,65^{++}\right)$et sur sa variation de 1979 à 1980 ; elles l'est encore en $1980\left(F=16,62^{++}\right)$mais la signification disparaît ensuite (tabl. 8). La figure 5 montre la réduction de la population de 1979 due au traitement, compensée partiellement de 1979 à 1980. Les liaisons avec les facteurs du milieu, non représentées, sont peu modifiées.

— L'indice de surpopulation «SURP» 1971 est étroitement lié à $Z_{1971}$ et à la variable «TOTAL», mais cette liaison décroît de façon importante avec $Z_{1972}$ puis avec $\mathrm{Z}_{19733}$ (tabl. 8). La figure 5 montre son incidence négative, très significative, sur les variations de populations de 1971 à 1973. Les relations avec le milieu, non représentées sur la figure 5, subsistent et leur forme est peu altérée (l'impact des expositions sud est un peu réduit). De la même façon, linfluence de la surpopulation en 1978 et 1979 a été mise en évdence. "SURP ${ }_{1978}$ et «SURP ${ }_{1979}$ sont très liés respectivement à $Z_{1978}$ et à $Z_{1979}$ et à la variable «TOTAL». La relation de «SURP $\gg_{1978}$ avec la variable $Z$ disparaît en 1979 ; celle de \&URP $»_{1979}$ diminue en 1980. Dans les deux cas la diminution de population est beaucoup plus importante sur les pins très infestés l'année précédente, mais la population s'accroît ensuite plus vite sur les pins qui avaient été surpeuplés et $\mathrm{Z}_{1982}$ est à nouveau très liée à ces deux indices.

Cette analyse rend compte de façon très illustrative de l'incidence des caractéristiques du milieu forestier sur les populations de processionnaire et met en évidence les modifications de leur effet au cours de la gradation. L'efficacité du traitement de 1979 est confirmée, mais l'analyse montre la reconstitution rapide des populations dans la zone traitée. L'impact des périodes de surpopulations sur la dynamique de l'insecte est également établie et la notion, depuis longtemps pressentie par les biologistes, de «pins pièges» subissant régulièrement de très fortes attaques est confirmée.

\subsection{Analyses factorielles}

\subsection{Analyse en composante principale}

On a fait appel à l'analyse en composantes principales pour obtenir une représentation plus synthétique des rapports entre les populations des différents cycles et de leurs relations avec le milieu avant le traitement. La population de 1979 n'a été introduite, comme les caractéristiques de l'environnement forestier, qu'en tant que variable supplémentaire ( $\$ 4.223$.).

La figure 6 résume les résultats obtenus pour tous les pins, très comparables à ceux des pins noirs seuls, par la métrique des covariances.

Près de 86 p. 100 de l'information du système est contenue dans les trois premiers axes principaux $(67,58$ p. $100,11,89$ p. 100 et 6,30 p. 100).

- Le premier axe est en corrélation d'autant plus étroite avec les années que linfestation est plus importante.

- Le second est corrélé négativement aux années 1971 et 1972 et à un moindre degré à 1974, positivement aux années 1975 à 1978, en particulier 1977 et 1978.

- Le troisième non représenté sur la figure 6 , apporte une information beaucoup plus limitée; il oppose essentiellement les populations de 1971 à 1982 et pourrait éventuellement traduire des relations de dépendance de la densité. 


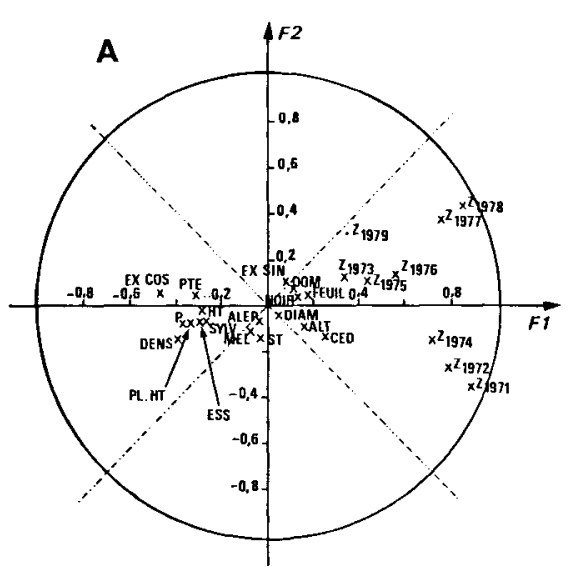

B

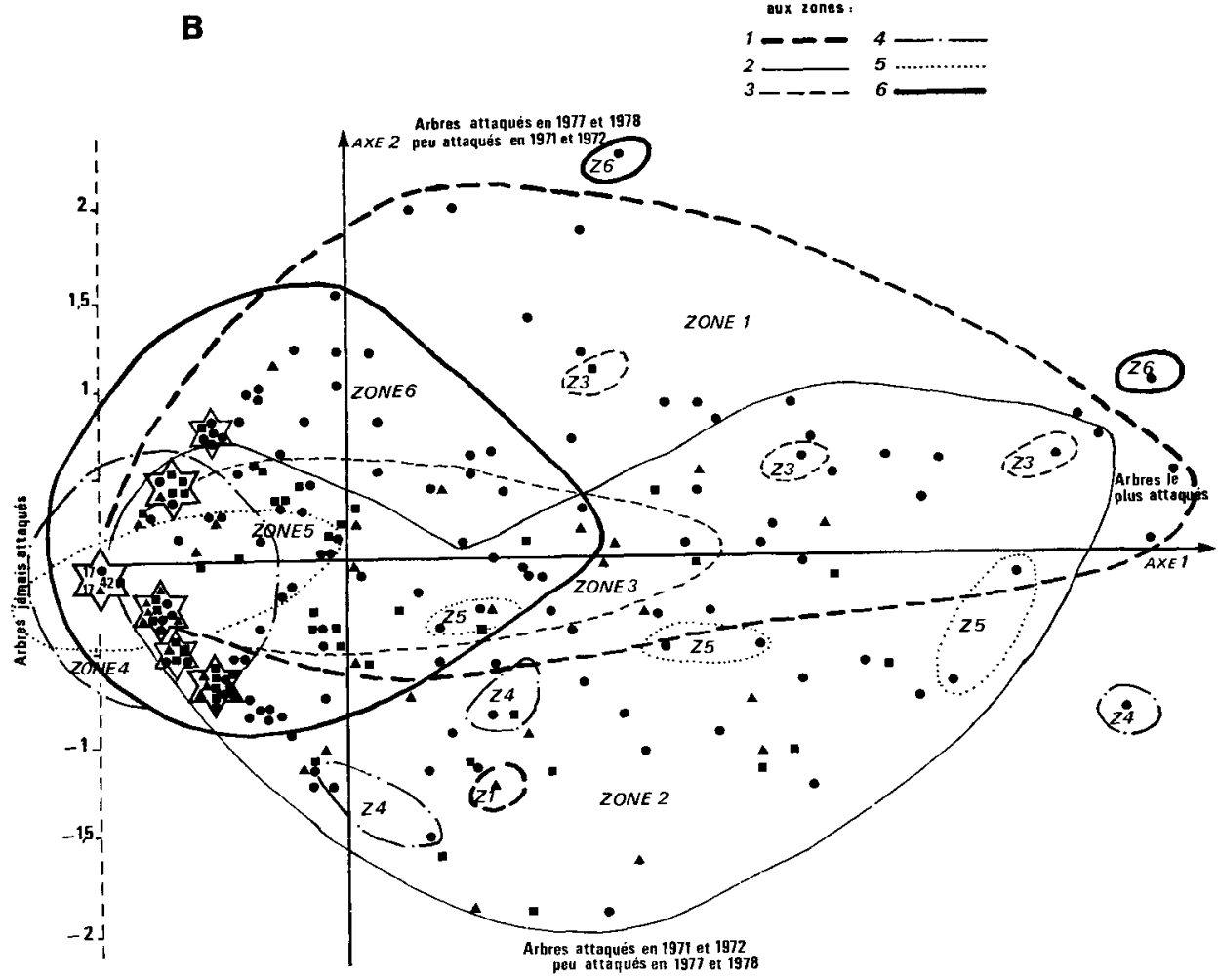

PTE : Pente

EX.SIN. : Sinus de I'exposition

ALT. : Altitude

UEL. : Mélange du pir noir àvec d'autres essenœes

Présence dans le peuplement.

NOTR - de pin noin

SYLV - de pin sytvestm

ALEP - de pin d'Alep

CED. - de cèdm

FEUIL. - de feuillus

ST: nombre d'étages

PL.HT. hauteur de l'étage principal DENS. densité du peuplemert

P. nombre de pins dans la placette

ESS. : essence du pin échantillon

HT. hauteur du pin échantillon

DIAM. diamètre du pin échantilion

DOM. : dominance

B 3 accumulation de points

Peuplements

- clair

- moyennement dense

- donse

Limites dos points oppartenan

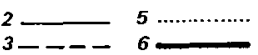

FIG. 6

Analyse factorielle en composantes principales.

A - Représentation des populations des différentes années et des variables supplémentaires suivant leurs corrélations avec les composantes principales des deux premiers axes principaux.

$B$ - Représentation des pins dans le plan des deux premiers axes principaux.

Principal components factor analysis.

A - Display of populations in various years and of additional variables according to their correlations with principal components of the two first principal axes.

$B$ - Display of pines on the plan formed by the two first principal axes. 
Lorsque l'on considère les populations des différentes années par rapport aux deux premiers axes principaux (fig. 6 A), il apparaît que les populations des cycles consécutifs se situent à proximité les unes des autres. Elles sont donc bien liées. En revanche les populations correspondant à la première phase d'évolution jusqu'en 1975 (qui correspond dans l'ensemble à une régression), s'opposent à celles correspondant à la période de «croissance» qui lui fait suite. Lopposition est maximale pour les populations importantes de 1971 à 1978, tandis que les faibles populations (1973 et 1975 ) ne jouent pratiquement aucun rôle. Ces faits sont confirmés par la représentation non figurée ici des variables $Z$ dans lespace formé par les 3 axes principaux.

La représentation des pins dans le plan des deux premiers axes (fig. $6 \mathrm{~B}$ ) montre la différence de répartition des populations durant ces deux phases. Ce sont essenticllement les populations importantes dos zones 1, 2 et 6 , exposées au sud, et à un moindre degré de la zone 3 qui sont différenciées par rapport à la première composante principale. La zone 2 (fortes attaques en 1971 et 1972) s'oppose aux zones 1, 6 et 3 (fortes attaques en 1977 et 1978) par rapport à la seconde. A l'exception de quelques peuplements clairs assez peuplis situés surtout en zone 5, dont les populations paraissent avoir un comportement très voisin de celles de la zone 2, les faibles populations des zones 4 et 5 (face nord) ne jouent aucun rôle. Sur la première composante, l'incidence négative des expositions nord et des peuplements denses sur limportance des populations est très apparente

Les corrélations des composantes principales correspondant aux deux premiers axes principaux avec les variables supplémentaires (fig. $6 \mathrm{~A}$ ) confirment la dépendance des populations vis-à-vis du milieu forestier : corrélations négatives de la première composante, qui traduisent l'effet défavorable des expositions nord (cosinus de l'exposition), de la densité et des variables qui lui sont liées (nombre de pins par placette, taille des pins et de l'étage principal), du pin sylvestre comme essence-hôte : corrélations positives avec l'altitude, la dominance et les caractéristiques des sites chauds et clairiérés (cèdres et feuillus). (es variables ne paraissent pas intervenir dans la distinction des deux phases gradologiques $\left(2^{\circ}\right.$ composante principale) mais leur position vis-à-vis de l'axe 1 , explique leur incidence différenciée au cours des périodes de croissance et de décroissance. La population de 1979 correspond bien à une réduction des effectifs. La même représentation avec le $3^{*}$ axe principal fait apparaître la liaison entre la variation de population de 1971 à 1972 et les expositions nord.

Le calcul avec la métrique des corrélations donne une plus grande importance aux faibles populations; il oppose en particulier les populations de 1973 (plus fortes populations dans la zone 2) et de 1975 (plus fortes populations dans la zone 1).

Cette analyse, tout en confirmant la liaison entre les populations des différents cycles et leurs relations avec le milieu, met donc en évidence des modifications importantes de leur répartition au cours de la gradation, avec deux périodes distinctes avant et après 1975. Toutefois, l'importance donnée dans ce phénomène aux différentes populations varie suivant la métrique employée. Bien que l'utilisation de la métrique des covariances se conçoive assez bien, les faibles populations n'étant constituées que de colonies isolées dont la répartition risque d'être aléatoire, il peut être dangereux de négliger ces dernières. L'analyse discriminante a été utilisée, pour préciser les différentes étapes de l'évolution des populations. 

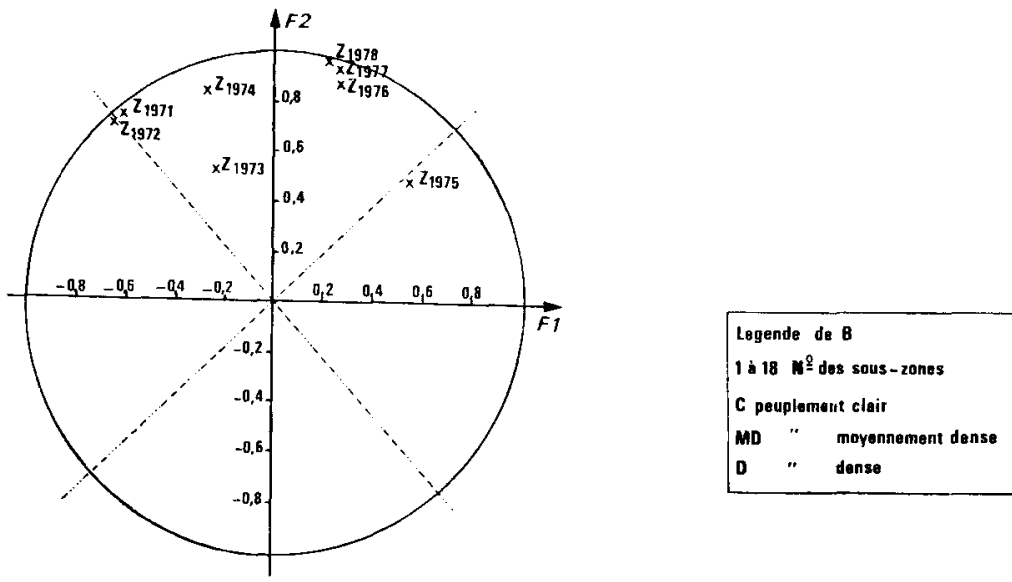

B

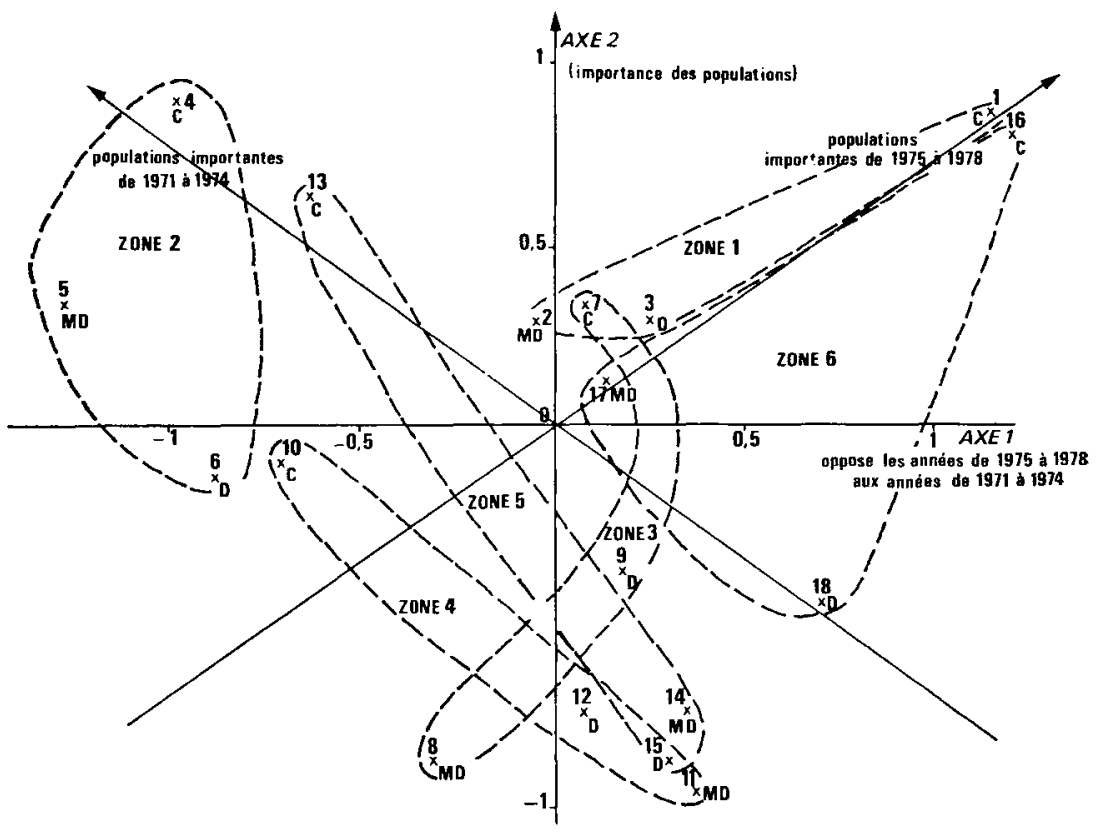

FIG. 7

Analyse discriminante.

A - Représentation des populations des différentes années

suivant leurs corrélations avec les variables discriminantes (niveau des sous-zones).

B - Représentation des sous-zones dans le plan formé par les deux premiers axes canoniques.

Discriminant analysis.

A - Display of populations in various years according to their correlations with discriminant variables (sub-zones level).

$B$ - Display of sub-zones on the plan formed by the two first cononical axes. 


\subsection{Analyse discriminante}

On a recherché par cette analyse, sur les variables $Z$, pour les pins noirs seuls ct pour l'ensemble des pins, les axes canoniques minimisant par projection sur ces axes les distances des pins de chaque sous-zone et maximisant les distances entre sous-zones (distance des centres de gravité des nuages de points). Le pouvoir discriminant do ces axes est testé par la statistique de Fisher.

Seuls les deux premiers présentent un $X \geq$ très significatif. Ces deux directions sont très discriminantes $\left(F=334^{++}\right.$et $\left.F=216^{++}\right)$pour l'ensemble des pins ; pour les pins noirs $\left(F=306^{++}\right.$et $\left.F=170^{+}\right)$, la première prédomine.

Pour l'ensemble des pins, le premıer axe est lié positivement aux années 1971 à 1974, négativement à 1975 et aux années suivantes. Le second présente avec les années des corrélations d'autant plus nettes que leur effectif est élevé; cette liaison est particulièrement marquée à partir de 1975.

Pour les pins noirs seuls, dont les corrélations avec les années sont représentées pour les valeurs moyennes par sous-zone sur la figure 7 , les axes sont encore mieux définis. Le premier oppose les années 1971 à 1974 aux années 1975 à 1978. Le second traduit exclusivement l'importance des effectifs des différentes années.

La figure 7 montre l'antagonisme entre les populations des deux phases évolutives centrées sur deux bissectrices differentes des deux premiers axes.

La position des différentes sous-zones dans le plan formé par les axes canoniques fait apparaître l'opposition entre la zone 2, plus peuplée de 1971 à 1974, et les zones 1 et 6 plus peuplées de 1975 à 1978 ; l'opposition est peu marquée pour les zones 3, 4 et 5 de la face nord. La situation des zones et sous-zones par rapport au deuxième axe confirme que le versant sud est le plus peuplé et que dans chaque zone les peuplements clairs sont les plus infestés. On peut également constater que les peuplements clairs du versant nord ont lendance à se comporter comme les zones I ct 6 (zone 3) et comme la zone 2 (zones 4 et 5). Des graphiques très voisins sont obtenus pour l'ensemble des pins.

Cette analyse confirme donc l'existence de deux périodes distinctes dans l'évolution des populations correspondant à une modification de leur répartition dans le massif. Les axes canoniques sont peu différents des axes principaux mais l'axe qui différencie ces périodes prend la plus grande importance. Le rôle des années est établi plus précisément et indépendamment de leurs effectifs. Il apparaît notamment que les populations résiduelles de 1975 ont une répartiton très différente de celle des années antérieures. Elle montre en outre, de façon très apparente, que l'évolution des populations est voisine dans les peuplements clairs de la face nord et dans les peuplements de la face sud, en particulier dans les peuplements clairs des zones 4 et 5 et dans les peuplements de la zone 2 .

\subsection{Discussion}

L’intérêt de ces différentes approches statistiques peut être comparé. Les corrélations simples constituent une première étape de l'analyse des données. Elles font 
apparaître de nombreuses relations confirmées par des analyses plus élaborées : liaison des populations avec l'environnement, rapports entre les populations des différents cycles, distinction de plusieurs phases dans leur évolution. Ces relations doivent être considérées avec prudence en raison des liaisons entre variables. Les corrélations entre facteurs du milieu et les tableaux de contingence peuvent en donner un début d'interprétation.

L'analyse de variance non orthogonale permet d'apprécier plus finement les rapports entre les populations et l'environnement. Il apparaît que les facteurs sylvicoles, les facteurs physiques et la densité des populations ont une action relativement indépendante, ce qui explique la cohérence des résultats de cette analyse avec les corrélations malgré les liaisons entre variables du milieu. L'exposition, la densité des peuplements et l'essence hôte ont, comme dans tous les massifs étudiés, un rôle déterminant. L'incidence de ces facteurs de même que celle de la taille des pins et de la dominance est vraisemblablement liée aux processus éthologiques (DEMOLIN, $1969 \mathrm{a}$ et b) : les femelles, qui se dirigent vers des silhouettes puis choisissent les arbres dont la forme des aiguilles convient au mieux à la ponte, colonisent préférentiellement les sites clairiérés, les pins d'une certaine taille et d'une certaine essence et les arbres non protégés par le couvert. Les chenilles recherchent l'ensoleillement des boisements clairs, des expositions sud et des arbres dominants et s'y développent mieux que dans d'autres sites, en outre elles s'enfouissent dans les clairières pour se nymphoser. L'altitude, de même, dans une certaine mesure, que la taille des pins par ses relations avec la densité, semblent avoir une action plus étroitement dépendante des conditions du massif. Cette analyse, développéc pour tenir compte des populations antérieures et tester l'effet des facteurs du milieu sur les variations de population fournit des résultats proches de ceux de lanalyse de covariance non orthogonale, mais formulés en fonction d'un modèle plus simple qui met clairement en évidence la forme des liaisons. Ces résultats confirment l'existence de deux phases dans l'évolution des populations, correspondant à un impact différent des populations antérieures et des caractéristiques forestières. Grâce à deux indices simples, lanalyse de variance non orthogonale a permis de tester l'incidence du traitement et des fortes densités sur la dynamique de l'insecte. L'effet du traitement est rapidement compensé dans la zone traitéc. Les arbres gravement infestés subissent ultérieurement une réduction de population plus importante que les autres pins, mais sont rapidement repeuplés; ils constituent par leur situation dans le massif (ou par d'autres caractéristiques individuelles de l'arbre) de véritables «pins pièges» toujours très attaqués.

L'analyse en composantes principales met clairement en évidence une première phase caractérisée par de hauts niveaux de population dans la zone 2, suivie d'une période de progression, caractérisée par un peuplement plus important des autres zones du versant sud. Elle confirme la dépendance des populations vis-à-vis des facteurs du milieu forestier. L'analyse discriminante précise ces modifications de la répartition des populations. Elle montre l'analogie du développement des populations dans la zone 2 et dans certaines sous-zones du versant nord. Celles-ci constituent par suite les meilleurs témoins pour représenter ce qu'aurait été l'évolution des populations de la zone 2 sans traitement. 


\section{Conclusion}

Une véritable compréhension de la dynamique des populations de processionnaire au Mont-Ventoux ne peut être obtenue sans confronter les mesures de population à des obscrvations biologiques détaillées. Toutefois, les dénombrements de nids ne permettent pas seulement dapprécier les fluctuations de populations. Ils apportent des éléments importants en ce qui concerne la fréquence des gradations, les relations entre l'insecte et la structure de l'environnement forestier, l'incidence des interventions humaines, les facteurs susceptibles d'avoir un rôle important dans la régulation des populations. Ces informations sont à prendre en considération lorsqu'on désire établir une prévision des infestations (prognose) ou limiter les dégâts par un aménagement judicieux des forêts.

Les méthodes d'estimation utilisées en Corse ont été transposées avec succès au Mont-Ventoux, malgré des populations parfois très basses et un nombre de points d'observation limité. Les marges d'erreur ont été suffisamment faibles pour permettre d'apprécier les fluctuations naturelles des populations et les conséquences d'une intervention chimique. L'échantillon permanent de pins, tout en apportant un gain de précision, permet une analyse beaucoup plus détaillée de l'évolution des populations. Par ailleurs, la valeur d'indices très simples et rapides à apprécier a été simultanément testée. Ces indices peuvent être utilisés sur de plus vastes territoires à des fins de surveillance et de prognose. La comparaison entre les recensements représentatifs du massif et les dénombrements effectués sur des surfaces limitées par les forestiers montre que les seconds ont fourni une information assez satisfaisante sur les fluctuations de populations. Ce résultat a déjà été appliqué par le C.E.M.A.G.R.E.F. pour la surveillance des populations et la prévision des attaques au niveau national. Au Ventoux, l'évolution des populations a pu être retracée depuis 23 ans. Les gradations sont plus ou moins cycliques et leur régularité est vraisemblablement perturbée par des facteurs aléatoires (climat, diapause). I_es six années de très faibles populations sont peut-être la conséquence du traitement à base de virus de 1959. Les effets de l'intervention de 1979 sont également mis en évidence.

Les relations entre l'importance des populations et les caractéristiques forestières peuvent être interprétées comme des conséquences de l'éthologie de l'insecte. Elles mériteraient d’être prises en considération dans la sylviculture dı massif. Une attention particulière devrait être accordée à la face sud, où un certain nombre d'opérations pourraient être entreprises pour limiter les foyers : traitements localisés, accroissement de la densité des peuplements en favorisant la croissance, réduction des clairières, amélioration de régénération sous couvert, diversification des essences. L'implantation des cèdres, beaucoup moins attaqués que les pins noirs et bien adaptés aux faces sud, devrait être encouragée. La révision du plan d’aménagement de la forêt de Malaucène (Office Natonal des Forêts, 1983) va en ce sens : des travaux d'amćlioration des peuplements et des régénérations sont prévus, ainsi que l'accroissement des surfaces boisées en cèdres.

La grande efficacité du diflubenzuron est démontrée. La destruction totale des populations de la zone traitée ne peut être confondue avec la régression générale observée par la suite dans le massif. Ces populations auraient vraisemblablement subi un accroissement important de 1978 à 1979, comme celles des peuplements 
clairs des zones 4 et 5 dont l'effectif a doublé. Par contre, la zone traitée cst rapidement repeuplée et l'interprétation d'une éventuelle incidence du traitement à plus long terme aurait nécessité une plus longue étude des populations naturelles.

L incidence des fortes populations sur la dynamique de linsecte est mise en évidence. Les phénomènes sous jacents (réduction quantitative et qualitative de la nourriture, modification de l'état physiologique des insectes, dispersion des adultes vers d’autres arbres) mériteraient d'être approfondis. L'évolution naturelle des populations jusqu’en 1978 présente d’abord une phase de régression, dans laquelle l'importance des populations de 1971 paraît être intervenue et durant laquelle les sites favorables à la processionnaire furent particulièrement dépeuplés; puis une phase de progression, caractérsée par un retour de plus en plus marqué au schéma habituel de répartition des populations. Durant ces deux périodes, on observe une distribution assez différente des populations entre les différentes zones du massif.

\title{
Remerciements
}

Nous tenons à remercier particulièrement l'O.N.F. d'Avignon pour les données sur les dénombrements parcelllaires de nids et le STEFECE pour les informations climatiques sur le Ventoux, ainsi que R. Riela, M. Gigout, C. Bricot, F. Goussard et G. Arretenu, techniciens de la station de biométrie de Nancy et de la Station de Zoologic forestière d’Orléans, pour l'aide qu'ils nous ont apportée dans la réalisation de ce travail.

\author{
Summary \\ Evaluation of the populations of pine processionary caterpillar \\ (Thaumetopoea pityocampa Schiff. Lepidopterae thaumetopoeidae) \\ in Mont-Ventoux, France
}

This article deals with the transposition of census methods by counting nests, used on processionary populations, from high-altitude forests of Corsica to afforestations of Mount Ventoux (Vaucluse).

Populations are numbercd by stratified random sampling on a 320 ha area, located at the western boundary of the moutain. Statistical multivariate analyses (correlation, non orthogonal variance and covariance analysis, principal components factor analysis, discriminant analysis) are carried out to study connections between populations and their forest surroundings, to estimate the impact of control operations and to account for the effect of some factors acting on population dynamics of the insect.

The interest of these different statistical analysis is compared. The sampling design allows to measure natural population fluctuations and shows the efficiency of a treatment with diflubenzuron. Representative estimates with regard to the whole area as compared with nests counting made in 3 plots by foresters of the "Office National des Forêts 》 led to the reconstruction of population evolution since 1959. Concerning forest factors, links between the population size on the one hand, the exposure, the woodland density, the host species on the other hand are confirmed. It is shown that the effect of these factors is general, whereas the result of other factors as elevation is more dependant on the particular characteristics of each forest. Two distinct periods are revealed in population evolution since 1970. These results support the importance of behavioural insect features upon population dynamics and suggest the effect of density dependant factors. 


\section{Références hibliographiques}

Antonioletti R., Baculat B., 1980. Caractérisation climatique du Mont Ventoux. I. Le Climat thermique. Avignon - Montfavet : I.N.R.A., Station de bioclimatologie, 67 p. Note tech., 81-1.

AuEr C., 1961. Ergebnisse zwölfjahriger quantitativer Untersuchungen der Populationsbewegungen des grauen Lärchenwicklers Zeiraphera griseana Hübner (= diniana Gućnée) im Oberangadin (1949/1960). Mitt. schweiz. Anst. forstl. Versuchswes., 37, 165-263.

Auer C., 1968. Erste Ergebnisse einfacher stochastischer Modelluntersuchungen ïber die Ursachen der Populationsbewegung des grauen Lärchenwicklers Zeiraphera diniana Gn. $(=$ Z. griseana $\mathrm{Hb}$.) im Oberengadin, 1949/1966. Z. angew. Entomol., 62, 202-235.

Bachacou J., Masson J.P., Miller C., 1981. AMANCE 81, Manuel de programmathèque statistique. Versailles : I.N.R.A., $516 \mathrm{p}$.

Cailliez F., Mallles J.P., Nakache J.P., Pages J.P., 1971. Analyse des données multidimensionnelles d'entrcprise. Paris : Cent. Et Econ, $t$ II, 113-186.

Cailliez F., Pages J.P., 1976. Introduction à l'analyse des données. Paris : SMASH, 616 p. Demolin G., 1969 a. Bioécologia de la Processionaria del pino, Thanmetopoea pityocampa Schiff. Incidencia de los factores climaticos. Bol. Serv. Plagas Forest., 23, 9-24.

Dfmolin G., 1969 b. Comportement des adultes de Thammetopoea pityocampa Schiff. Dispersion spatiale, importance économique. Ann. Sci. for., 26 (1), 81-102.

Demolin G., 1974. Incidence du climat sur les gradiations de la Processionnaire du pin. Thaumetopoea pityocampa Schiff. Recherches d'éléments ou d'indices sur les potentialités négatives ou positives des populations Prognose. C.R. de fin de contrat D.G.R.S.T. $\mathrm{n}^{\circ} 70.021 .74,17 \mathrm{p}$.

Demolin G., Millet A., 1981. Essais dinsecticides contre la Processionnaire du pin (Thaumetopoea pityocampa Schiff.). Action comparative de différentes spécialités commerciales, Bactospéine, Dipel, Thuricide et Dimilin. Ann. Sci. for., 38 (3), 389-404.

Dusaussoy G., Geri C., 1969. Etude des fluctuations du niveau de population de la Processionnaire du Pin dans la vallée du Niolo en Corse. Ann. Sci. for., 26 (1), 103-125.

GerI C., 1971. Etude du niveau de population de Thaumetopoea pityocampa Schiff. dans la vallée du Niolo en Corse. Notes préliminaires sur les cycles biologiques (1965-1966 et 1967-1968). Ann. Zool. Ecol. Anim., 2 (2), 67-87.

Geri C., 1980 a. Application des méthodes d'études démécologiques aux insectes forcstiers, cas de Diprion pini L. (Hymenoptera Diprionidae) et Thametopoea pityocampa Schiff. (Lepidoptera, Thametopeïlae). Dynamique des populations de processionnaires du pin Thaumetopoea pityocampa dans lîle de Corse. Thèse Etat. Orsay Univ. Paris Sud. $249 \mathrm{p}$.

Geri C., 1980 b. Etudes sur les populations de Processionnairc du Pin (Thatmetopoea pityocampa Schiff., Lepidoptera Thametopoeidae). P. GRISON, In \& Etudes Ecologiques et Ethnologiques dans le Niolo». Ed, Eco. Mediterr., 6, 151-172.

Geri C., 1983. Dynamique de la Processionnaire du pin dans la valléc du Niolo, en Corse au cours de cycles 1965-1966, 1967-1968, 1969-1970. Rôle de certains caractères du milien forestier. Ann. Sci. for., 40 (1), $111-137$.

GRISON P., 1969. Reflexiones sobre la utilizacion de Smithiavirus pityocampae VAGO en la lucha microbiologica contra Thaumetopoea pityocampa Schiff. Bol. serv. plagas for., 12 (24), 105-112.

Grison P., Vago C., Maury M., 1959. La lutte contre la Processionnaire Pin, Thaumetopoea pityocampa Schiff. dans le massif du Ventoux. Essai d'utilisation pratique d'un virus spécifique. Rev. for. fr., 5, 353-370.

MorRIS R.F., 1963. Predictive equations based on key factors. Men. Soc. entomol. Can., 32, 16-21.

O.N.F., 1983. Forêt domaniale de Malaucene. Procès verbal de révision d'aménagement 1984, 2007, 13 p.

Pinguet A., Samie C., 1980. Eléments sur le climat pluvio-thermique du Valdo-Niello, P. Grison, In «Etudes Entomologique et Ethnologique dans le Niolo». Ed. Ecol. Mediterr., 6, 43-99. 